\title{
DIII-D Data for Modeling the Scrape-Off-Layer Plasma
}

\author{
D. N. Hill \\ D. Buchenauer \\ T. N. Carlstrom \\ J. Ferron \\ M. Rensink
}

December 4, 1989

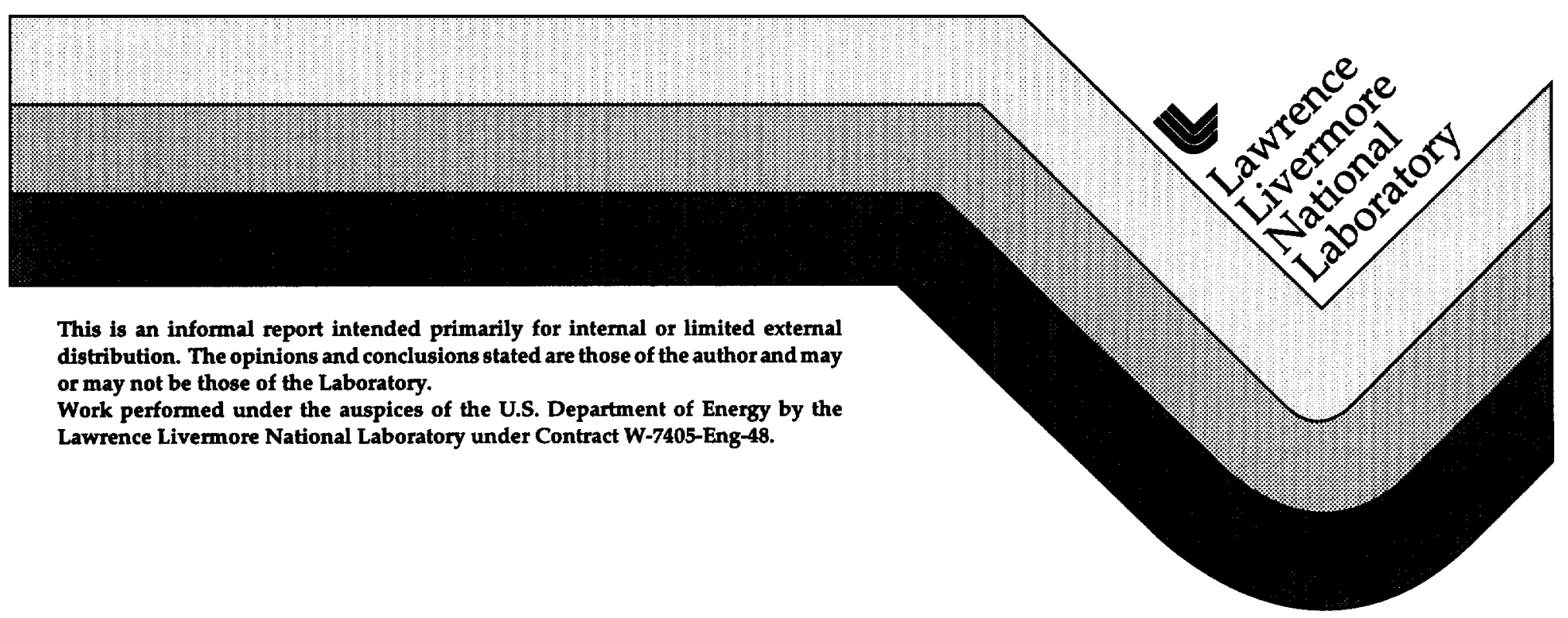




\section{DISCLAIMER}

This document was prepared as an account of work sponsored by an agency of the United States Government. Neither the United States Government nor the University of California nor any of their employees, makes any warranty, express or implied, or assumes any legal liability or responsibility for the accuracy, completeness, or usefulness of any information, apparatus, product, or process disclosed, or represents that its use would not infringe privately owned rights. Reference herein to any specific commercial product, process, or service by trade name, trademark, manufacturer, or otherwise, does not necessarily constitute or imply its endorsement, recommendation, or favoring by the United States Government or the University of California. The views and opinions of authors expressed herein do not necessarily state or reflect those of the United States Government or the University of California, and shall not be used for advertising or product endorsement purposes.

This report has been reproduced directly from the best available copy.

Available to DOE and DOE contractors from the Office of Scientific and Technical Information P.O. Box 62, Oak Ridge, TN 37831

Prices available from (615) 576-8401, FIS 626-8401

Available to the public from the National Technical Information Service

U.S. Department of Commerce

5285 Port Royal Rd.,

Springfield, VA 22161 


\section{Table of Contents}

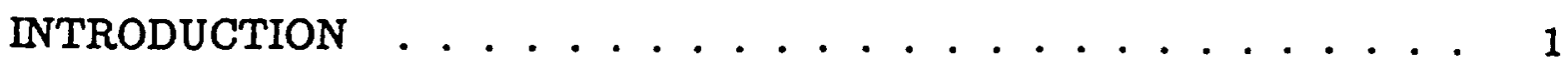

I. DIAGNOSTICS .................... 4

II. CHARACTERIZATION OF H-MODE PLASMAS . . . . . . . 4

II.A. Divertor Plasma Parameters for the Quiescent Phase . . . . . 7

II.B. Divertor Parameters for Quasi-Stationary H-Modes . . . . . . . 10

III. DIVERTOR HEAT-FLUX ASYMMETRIES . . . . . . . . . . . . 19

IV. I-MODE SOL DATA MODELING . . . . . . . . . . . 25

IV.A. Scaling Data for L-Mode Discharges . . . . . . . . . . . 25

IV.B. Modeling the L-Mode SOL Plasma Using B2 . . . . . . . . . 33

V. SUMMARY AND NEAR-TERM PLANS . . . . . . . . . . . . . 38

REFERENCES .......................... 41

ACKNOWLEDGMENTS .................. . . 43

\section{List of Figures}

1. DIII-D cross section with divertor diagnostics . . . . . . . . . 5

2. Typical quasi-stationary $\mathrm{H}$-mode discharge with frequent ELMs . . . . 6

3. Typical discharge parameters for an $\mathrm{X}$-point scan across the divertor Langmuir probes . . . . . . . . . . . . . . . . . . . . . 8

4. Profiles of divertor plasma parameters at outboard target . . . . . . . 9

5. Divertor heat flux for quasi-stationary $\mathrm{H}$-mode . . . . . . . . . . . . 12

6. Divertor heat flux during a typical ELM . . . . . . . . . . . . 13

7. Radial profiles of divertor heat flux between and during ELMs . . . . . 14 
8. Radial profiles of divertor particle flux, top, $\mathrm{H}_{\alpha}$ emission at outside intercept, middle, and program voltage for X-point sweep, bottom . . . 16

9. Power balance for steady-state $\mathrm{H}$-mode discharges . . . . . . . . 18

10. Energy flux across the separatrix as a function of distance along the separatrix calculated using the B2 code . . . . . . . . 20

11. Typical parameters for $\mathrm{L}$-mode and $\mathrm{H}$-mode discharge showing large divertor heat flux asymmetry . . . . . . . . . . .

12. Divertor heat flux profiles for two similar plasmas except for a reversal of toroidal field so that the ion $\nabla \mathrm{B}$ drift is away from instead of towards the X-point . . . . . . . . . . . 23

13. Divertor heat flux for double-null divertor discharge . . . . . . 26

14. Divertor heat flux profiles versus time for discharges with upper and lower single-null configurations . . . . . . . . . . . 27

15. Variation of edge plasma pressure with NBI heating power for I-mode discharges . . . . . . . . . . . . . . . 29

16. Radiative losses versus power for $\mathrm{L}$-mode discharges . . . . . . . . 30

17. Total integrated divertor power versus total input power for $\mathrm{L}$-mode discharges . . . . . . . . . . . . . 31

18. Discharge characteristics for $L$-mode $\mathrm{X}$-point sweep ～. . . . . . . . 32

19. Outboard divertor heat flux profiles calculated using the B2 code . . . . 35

20. Comparison of calculated versus measured midplane density profiles . . 36

\section{List of Tables}

I. Power Balance for H-Modes with ELMs . . . . . . . . . . . . . 17

II. Heat Flux Asymmetry Studies . . . . . . . . . . . . 21

III. Transport Coefficients for Lr-Mode Plasmas . . . . . . . . . . . 37 


\title{
DIII-D DATA FOR MODELING THE SCRAPE-OFF-LAYER PLASMA
}

\author{
D.N. Hill , D. Buchenauer, T.N. Carlstrom, J. Ferron, M. Rensink
}

December 4, 1989

\section{Introduction}

We are in the process of assembling a database of edge and divertor plasma parameters suitable for use in benchmarking various 2D models of the scrape-offlayer (SOL) plasma. Also, we are using the Braams B2 code to derive transport coefficients for the edge plasma. In parallel, work is starting on an upgrade to the B2 code that includes parallel current flow and $E \times B$ drifts. These efforts are directed at increasing the confidence level of models of the tokamak edge plasma so that we can predict the effect of planned upgrades to DIII-D (e.g., the Advanced Divertor Program) and the performance of next generation machines such as CIT or ITER, where initial design studies show that plasma conditions at the divertor targets can have a large impact on the lifetime and cost of the machine. This report summarizes our recent progress in characterizing the DIII-D SOL plasma and in modeling these data with the the $\mathrm{B} 2$ code $^{1}$.

Our measurement effort at DIII-D is focussed on three different operating regimes: NBI heated L-mode, NBI heated quiescent H-mode(no Edge Localized Modes-ELMs), and the so-called "steady-state" H-mode, which has frequent ELMs. L-mode is of interest primarily because all of the plasma parameters achieve steady state and this is easier to measure and model. The quiescent $\mathrm{H}$-mode observed after 
the L-to-H transition can last for relatively long periods ( 0.5 to $0.9 \mathrm{sec})$, depending on the initial plasma density, discharge current, and input power. Although relatively easy to diagnose, an $\mathrm{H}$-mode without-ELMs is probably not directly relevant. to ITER unless density control can be achieved. Otherwise, the density and radiative losses climb steadily until ELMs appear or the plasma returns to L-mode confinement. Presently, DIII-D steady-state H-mode probably represents the best plasma to scale to ITER. The time-averaged energy confinement is nearly as good as that for the quiescent $\mathrm{H}$-mode and it does not have the problem of impurity accumulation. However, it is difficult to diagnose because the ELMs produce periodic bursts of particles and power onto the divertor targets which are as much as ten times above the background level, and there are few time-dependent codes with which to model such transient data.

We have so far obtained the scaling of divertor heat flux with input power for ohmic, L-mode, and quasi-stationary $\mathrm{H}$-mode plasmas and used these data for power balance calculations. In quasi-stationary $\mathrm{H}$-modes we find that about $30 \%$ of the power reaches the divertor targets between ELMs; the ELMs themselves, on a time-averaged basis, carry out less. As reported earlier ${ }^{2}$, the heat flux to the divertor targets is poloidally asymmetric (greatest at the outside strike point), but more recent operation with reversed $\mathrm{B}_{\mathrm{T}}$ (ion $\nabla \mathrm{B}$ drift away from the divertor) shows the opposite behavior. This is consistent with the work of Hinton and Staebler ${ }^{3}$, which emphasizes the importantance of particle convection to the energy transport across the separatrix. Data from double-null (DN) divertor operation also shows what appears to be particle-drift effects since, in $\mathrm{H}$-mode, we find that while the total integrated heat flux to the targets can be balanced between the upper and lower divertors, there remains an almost 2:1 up/down asymmetry in the peak heat flux (greatest on the divertor in the ion $\nabla B$ drift direction-as predicted by Staebler ${ }^{4}$ ).

New Langmuir probe measurements at the outboard divertor target show that $\mathrm{T}_{e}$ rises with increasing neutral-beam injection (NBI) heating, up to about $45 \mathrm{eV}$ in $\mathrm{L}$-mode with $\mathrm{P}_{\mathrm{NBI}}=4.5 \mathrm{MW}$. In $\mathrm{H}$-mode, the peak electron temperature drops by only about $25 \%$, to about $35 \mathrm{eV}$. Following the H-mode transition the electron temperature and density profiles become narrower and the peak values shift, from being located outside, to inside of the calculated separatrix intercept (to the so-called "private region"). This shift may be the result of changes in the X-point magnetic topology due to thermoelectric currents in the SOL plasma. 
Our recent modeling efforts have focussed on (1) upgrading the standard B2 code to more more accurately simulate the DIII-D single-null geometry, (2) selecting a set of SOL plasma transport coefficients consistent with the measured edge profiles, and (3) testing the ability of the code to predict the observed power scaling of the divertor heat flux. We found that allowing diffusion into the private region significantly lowered the peak divertor heat flux and electron temperature and produced profiles in better agreement with the measurements (peaked off the separatrix). However, adding the effect of the poloidal field aull (allowing the connection length to the divertor to grow longer near the separatrix), yielded little change in the calculated divertor profiles. We examined four sets of SOL transport coefficients $\left(\chi_{i, e}, D_{\perp}\right.$, and $\left.v_{\text {conv }}\right)$, including three ITER reference cases; the best agreement between the calculated profiles and the experimental data was obtained with $\chi_{\mathrm{e}}=4 . \mathrm{m}^{2} / \mathrm{s}, \chi_{i}=0.2 \mathrm{~m}^{2} / \mathrm{s}, D_{\perp}=2.0 \mathrm{~m}^{2} / \mathrm{s}$, and $v_{\text {conv }}=-40 . \mathrm{m} / \mathrm{s}$ for $\mathrm{H}$-mode. Using these coefficients, we have calculated the expected inboard/outboard divertor heat-flux asymmetry and compared it to DIII-D data. The predicted value is too low and, more importantly, the predicted scaling with input power does not agree with the measured scaling. That is, the B2 code underestimates how the power flow to the outer divertor increases with input power. We are therefore pursuing upgrades to the code that could correct this result, such as adding poloidally localized losses across the separatrix, parallel current flow in the SOL, and thermoelectric $\mathbf{E} \times \mathbf{B}$ drift effects.

The remainder of this report is organized as follows. Section I contains a brief description of the diagnostics available for characterizing the SOL plasma. In Section II we present our measurements of the SOL parameters for $\mathrm{H}$-mode plasmas. This includes data showing how the divertor plasma parameters $\left(\mathrm{n}_{e}(r), \mathrm{T}_{e}(r)\right.$, and $\mathrm{Q}(r)$ ) vary from ohmic to $\mathrm{L}$-mode to $\mathrm{H}$-mode, and power balance for quasi-stationary $\mathrm{H}$-mode plasmas. Section III covers divertor-target heat-flux asymmetries for double and single null operation with forward and reversed toroidal field. In Section IV we show the scaling of L-mode parameters with neutral beam power, and Section V concludes with a summary of the results obtained from the Braams B2 SOL simulation code. 


\section{Diagnostics}

A cross section of DIII-D showing the relevant diagnostics appears in Fig. 1. There are several diagnostics which are capable of measuring plasma parameters in the SOL and divertor region. Edge density and temperature profiles are obtained from the Thomson scattering system. Profiles along the Thomson chord are mapped to the minor radius at the midplane using the ENERGY code 5 . A moveable Langmuir probe at the midplane can measure electron temperature and density profiles in the outermost part of the SOL ( $\geq 3 \lambda_{n}^{H-m o d e}$ ) on a shot-by-shot basis. Time dependent heat flux profiles at both the upper and lower divertor targets are obtained from two infrared (IR) TV cameras. Plasma density and temperature at the outermost divertor target can be measured with two prototype single-tip Langmuir probes ( $4 \mathrm{~mm}$ dia. graphite similar to the JET design ${ }^{6}$ ) supplied by SNLL. Profiles are obtained by sweeping the strike point across the probes during a single shot. Also, there are arrays of $\mathrm{H}_{\alpha}$ photodiodes and TV cameras viewing the divertor region to measure the profile of $\mathrm{H}_{\alpha}$ emission.

Our edge diagnostics capability will be greatly expanded in the near future when collaborators on the Advanced Divertor Program ${ }^{7}$ (GA, LLNL, SNLA, SNLL, and UCLA) bring new hardware to DIII-D. UCLA will install two fast-stroke Langmuir probes starting in June 1990. These will be able to sample the SOL plasma all the way in to the separatrix for NBI heated L-mode and H-mode plasmas. A lithium-beam diagnostic from GA (presently unfunded) could give additional profile information in this region. ORNL will install a visible spectrometer to view impurity emission from the divertor region. SNLL, in collaboration with LLNL and GA, will upgrade the full set of 20 tips in the divertor Langmuir probe poloidal array so that we can measure simultaneously the plasma parameters at both the inner and outer strike points.

\section{Characterization of $\mathrm{H}$-mode Plasmas}

The basic features of the H-mode are illustrated in Fig. 2, which shows data from a 1.25 MA deuterium plasma discharge heated by $7 \mathrm{MW}$ of hydrogen NBI. The H-mode transition occurs approximately $50 \mathrm{msec}$ after the start of NBI and is characterized by a sudden drop in divertor $\mathrm{H}_{\alpha}$ emission (trace d) and a rapid density buildup (c). In this case, a quasi-stationary state with frequent ELMs and relatively 


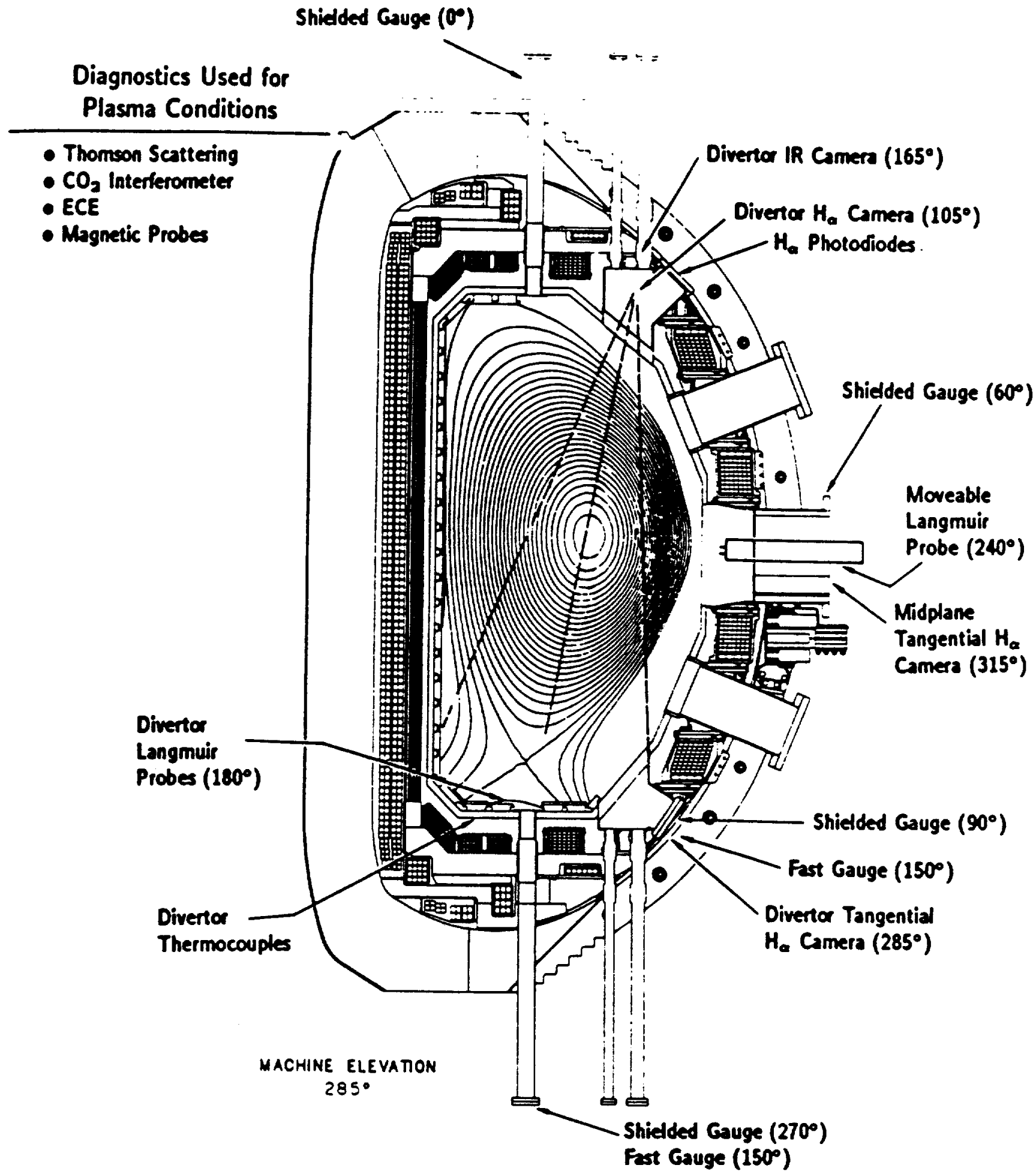

Fig. 1. DIII-D cross section with divertor diagnostics. 


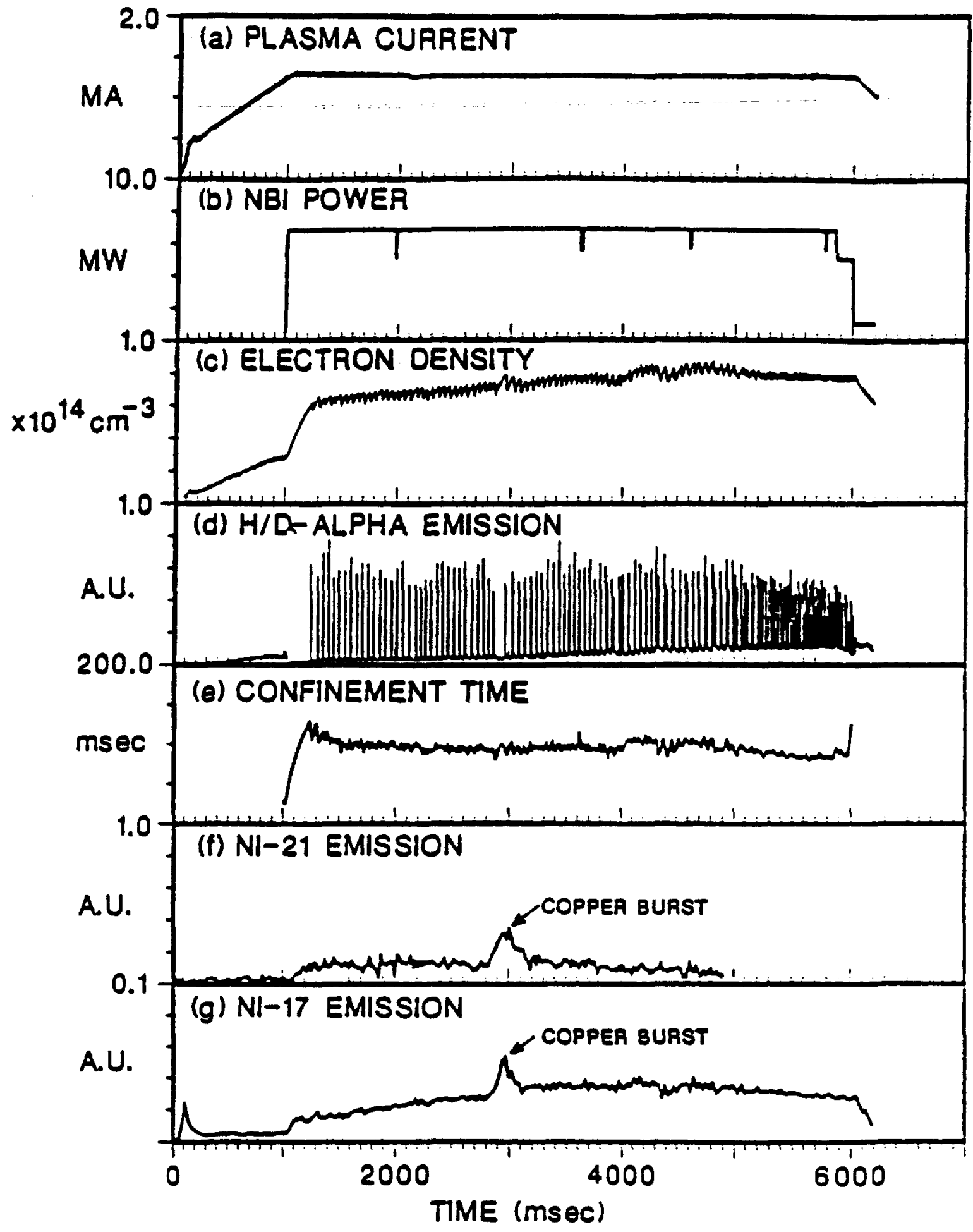

Fig. 2. Typical quasi-stationary H-mode discharge with frequent ELMs. 
constant density is achieved following the initial $200 \mathrm{msec}$ quiescent period. The confinement time (e) in this quasi-stationary phase is within the range previously reported $^{8}$ for DIII-D. We will now consider each of these phases separately.

\section{A. Divertor Plasma Parameters for the Quiescent Phase}

We have recently measured the divertor plasma parameters at the outboard strike point using the two prototype Langmuir probes supplied by SNLL. Profiles of local plasma density and temperature were obtained by moving the $\mathrm{X}$-point radially so that the separatrix intercept was stepped across the probe locations on a shotby-shot basis during a series of similar discharges. In this way we could study how the divertor plasma conditions evolved going from ohmic to L-mode, and then to $\mathrm{H}$-mode. These spatial scans were repeated at two plasma currents (1.2 and 1.6 MA) and with a toroidal field of 2.1 Tesla. H-mode was obtained about $250 \mathrm{msec}$ after the start of NBI $\left(D^{\circ} \rightarrow D^{+}\right)$, and the quiescent period lasted over $400 \mathrm{msec}$, as shown in Fig. 3. The initial target density at $1.25 \mathrm{MA}$ was $2.5 \times 10^{13} \mathrm{~cm}^{-3}$ and during the $\mathrm{H}$-mode rose to $7 \times 10^{13}$ just before the first ELM. At the same time the radiative losses steadily increased from $20 \%$ to $60 \%$ of the input power while the total power to the divertor targets remained near the $20 \%$ level.

The changes in the divertor plasma parameters resulting from the transition to $\mathrm{H}$-mode confinement are apparent in the profiles of electron density, temperature, and equivalent heat flux shown in Fig. 4. The heat flux is inferred from the Langmuir probe data using $Q_{\perp}=7 \mathrm{~T}_{e} \Gamma_{\perp, \mathrm{i}}$ where $\Gamma_{\perp, \mathrm{i}}$ is the ion flux normal to the divertor surface, not the flux along the field lines. The abscissa is the distance between the probe tip and the separatrix intercept as determined from the EFITD code ${ }^{9}$ : negative values correspond to the private region below the $\mathrm{X}$-point. Two features are clear. First, the profiles narrow considerably following the $\mathrm{H}$-mode transition while the peak temperature and density do not change very much $\left(45 \mathrm{eV}\right.$ and $3 \times 10^{13}$ to $35 \mathrm{eV}$ and $2.3 \times 10^{13}$ ). This is consistent with previous measurements ${ }^{10}$ of the midplane plasma profiles that show little change in $\mathrm{T}_{e}$ or $\mathrm{n}_{e}$ on the separatrix following the $\mathrm{H}$-mode transition. The behavior of the heat flux inferred from $n_{e}$ and $T_{e}$ is qualitatively similar to the direct measurements with infrared thermography, but the absolute values are a factor of four too large (possibly due to the effect of current flow on 


\section{QUIESCENT DD H-MODE DISCHARGE}

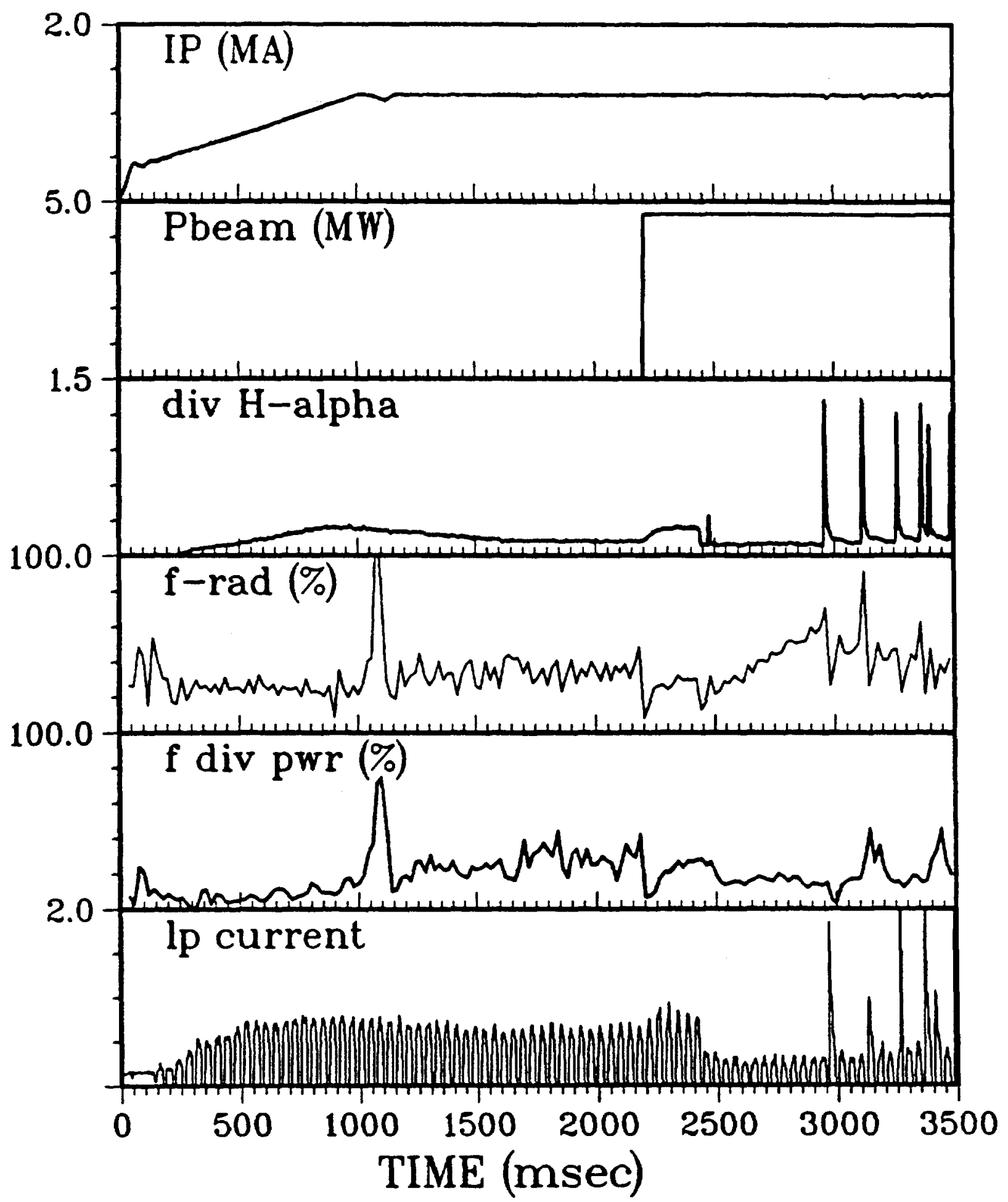

Fig. 3. Typical discharge parameters for an $X$-point scan across the divertor Langmuir probes. Probe current is labeled "Ip current". The fraction of input power reaching the divertor is "f div pwr" and the fraction radiated is "f-rad". 


\section{DIVERTOR PLASMA PROFILES Ohmlc, $L-m o d \theta$, and $H$-mode}
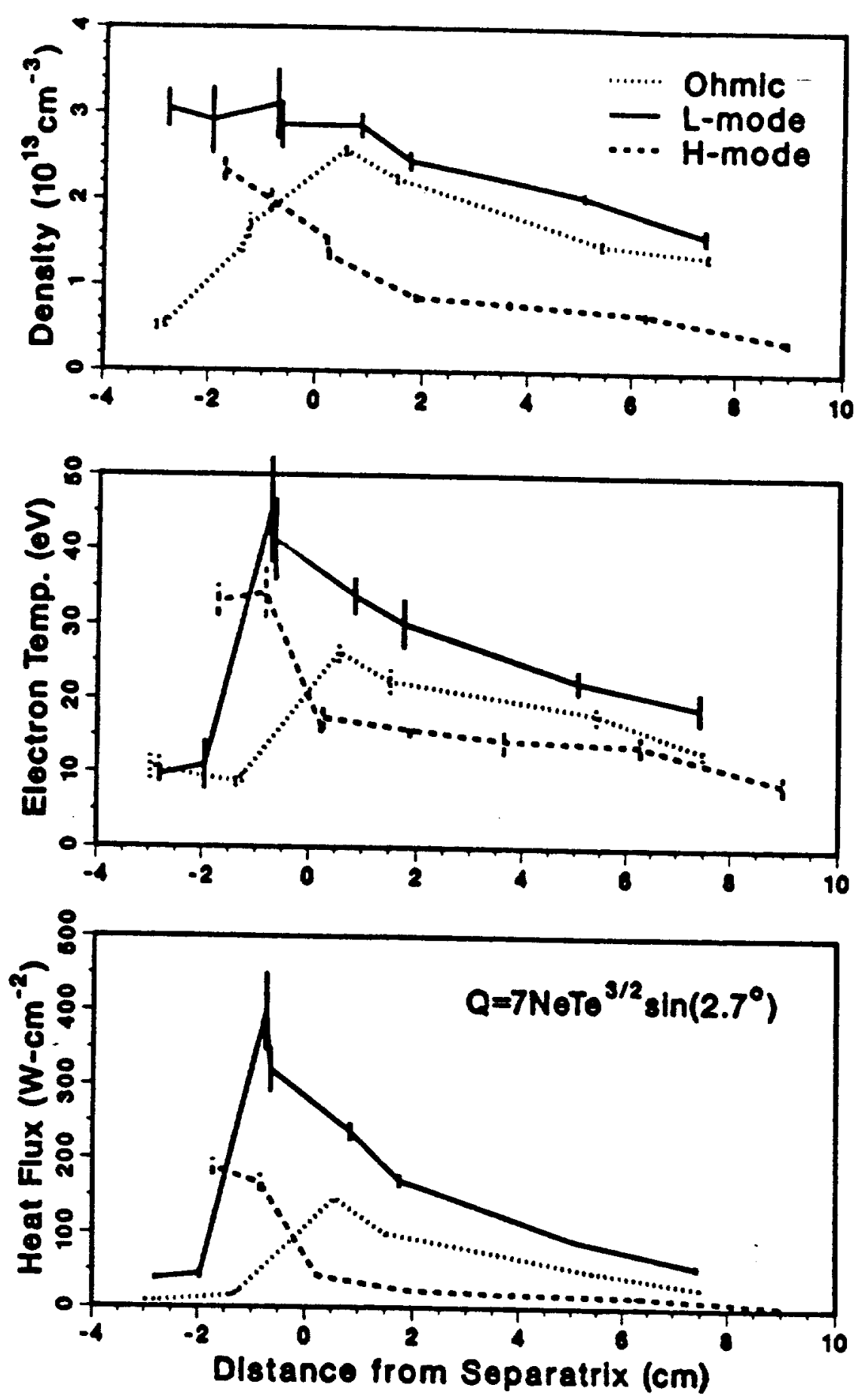

Fig. 4. Profiles of divertor plasma parameters at outboard target. Abscissa is the horizontal distance from the separatrix in $\mathrm{cm}$. Negative values are in the private region. 
the plasma sheath). The narrowing of the profiles implies that a sharp reduction of perpendicular transport in the SOL plasma has occurred.

The second feature of the quiescent $\mathrm{H}$-mode divertor plasma profile data is that the location of the peak temperature and density has shifted to inside the apparent separatrix intercept, as opposed to being outside for the ohmic and L-mode phases of the discharge. Such an inward shift following the H-mode transition has also been reported for the divertor heat flux, both for DIII- $D^{2}$ and ASDEX ${ }^{11}$. We have previously ${ }^{12}$ examined several sources of random and systematic error in the MHD calculation of the intercept location and concluded that they could not account for more than $\pm 0.5 \mathrm{~cm}$ shifts. Harbour has postulated ${ }^{6}$ that parallel current flow in the SOL, due to inboard/outboard electron temperature and divertor-plasma sheath asymmetries, changes the magnetic topology of the $\mathrm{X}$-point so that the actual intercepts are different than those calculated from EFITD. Stambaugh has previously calculated ${ }^{13}$ that current densities of the order of $90 \mathrm{kA} / \mathrm{m}^{2}$ can change the flux-surface crossing angle at the null by about $10^{\circ}$, and this would alter the location of the intercepts by approximately $2.5 \mathrm{~cm}$. At present, we do not have a direct measure of the net current flowing in the SOL plasma, but we are working to see if an existing tangential TV view of the $\mathrm{X}$-point region can detect such effects.

\section{B. Divertor Parameters for Quasi-stationary H-modes}

Casual inspection of $\mathrm{H}$-mode discharges such as the one shown in Fig. 2 suggest that the ELMs are responsible for clamping the the buildup of density and stored energy. A careful analysis by $P$. Stott ${ }^{14}$ has shown that both ELMs and radiative losses can limit the $\mathrm{H}$-mode confinement time in DIII-D. At modest input power, ELMs occur infrequently and have a relatively large amplitude ( $\geq 10 \%$ of $E_{\text {tot }}$ lost per ELM); between the ELMs the radiative losses can grow to be as large as $70 \%$ of the input power. At higher power, the ELM frequency increases and the amplitude falls ${ }^{15}$. The ohmic target plasma density can also influence the frequency, as can the $\mathrm{X}$-point location (higher $\mathrm{X}$-point generally gives fewer ELMs).

The observed scaling of ELM frequency in DIII-D is consistent with the picture that ELMs result when the edge pressure gradient exceeds the stability limit for ideal ballooning modes ${ }^{16,17}$, which is proportional to $S / q_{95}^{2}$, where $S$ is the edge magnetic 
shear and $g_{95}$ the safety factor at the $95 \%$ flux surface. Thus, higher NBI power drives the edge pressure up to the stability limit faster and so increases the ELM frequency. Conversely, higher plasma current increases the stability limit and so the ELM frequency drops because it takes longer to raise the edge pressure to the critical value. Ozeki ${ }^{18}$ has shown that varying the $\mathrm{X}$-point position can also change the edge stability and consequently the ELM characteristics.

The rapid ELMs observed during the steady-state H-mode strongly modulate the amplitude and poloidal distribution of the heat flux onto the divertor targets. We have recently begun to study the power flow to the divertor for such discharges; previously ${ }^{12}$ we examined only the initial quiescent $H$-mode period up to the first ELM, and treated the ELMs separately. Now, however, we have modified our data analysis ${ }^{2}$ so that the average of the heat flux to the divertor targets between ELMs may be obtained. Fig. 5 shows the time history of the total power $\left(\int 2 \pi R \cdot P(R) d R\right)$ to the divertor targets, as measured by the infrared TV camera, for one of these discharges operating in the lower single-null configuration. The divertor heat flux profile is included in (b), where the separatrix intercepts are indicated by the dashed vertical lines, and the $5 \mathrm{~cm}$ full width at the $1 / e$ points corresponds to about a $1 \mathrm{~cm}$ scrape-off width for the parallel heat flux at the plasma midplane. The large inboard/outboard asymmetry shown here-a factor of five in peak and three in total integrated power-develops only after the onset of rapid ELMs, which suggests that such ELMs also have a residual detrimental effect on the energy confinement during the quiescent $\mathrm{H}$-mode periods.

The peak heat flux onto the divertor targets during ELMs is about 10-20 times the value between the ELMs, but the duration of the burst is very short-typically less than $4 \mathrm{msec}$. The time history of the total divertor-target heat flux during a typical ELM is plotted in Fig. 6, along with the intensity of the $\mathrm{H}_{\alpha}$ emission from the same regions. The heat pulse is much shorter than the $\mathrm{H}_{\alpha}$ burst presumably because parallel heat conduction is much faster than the convective loss of ions to the divertor target. Also, unlike the heat flux during the quiescent periods, the flux during ELMs is larger on the inside target than on the outside, as shown in Fig. 7. Whether or not this is due to some toroidal mode structure is not known, but it suggests that the high losses due to ELMs are not simply the result of a return to L-mode confinement. 

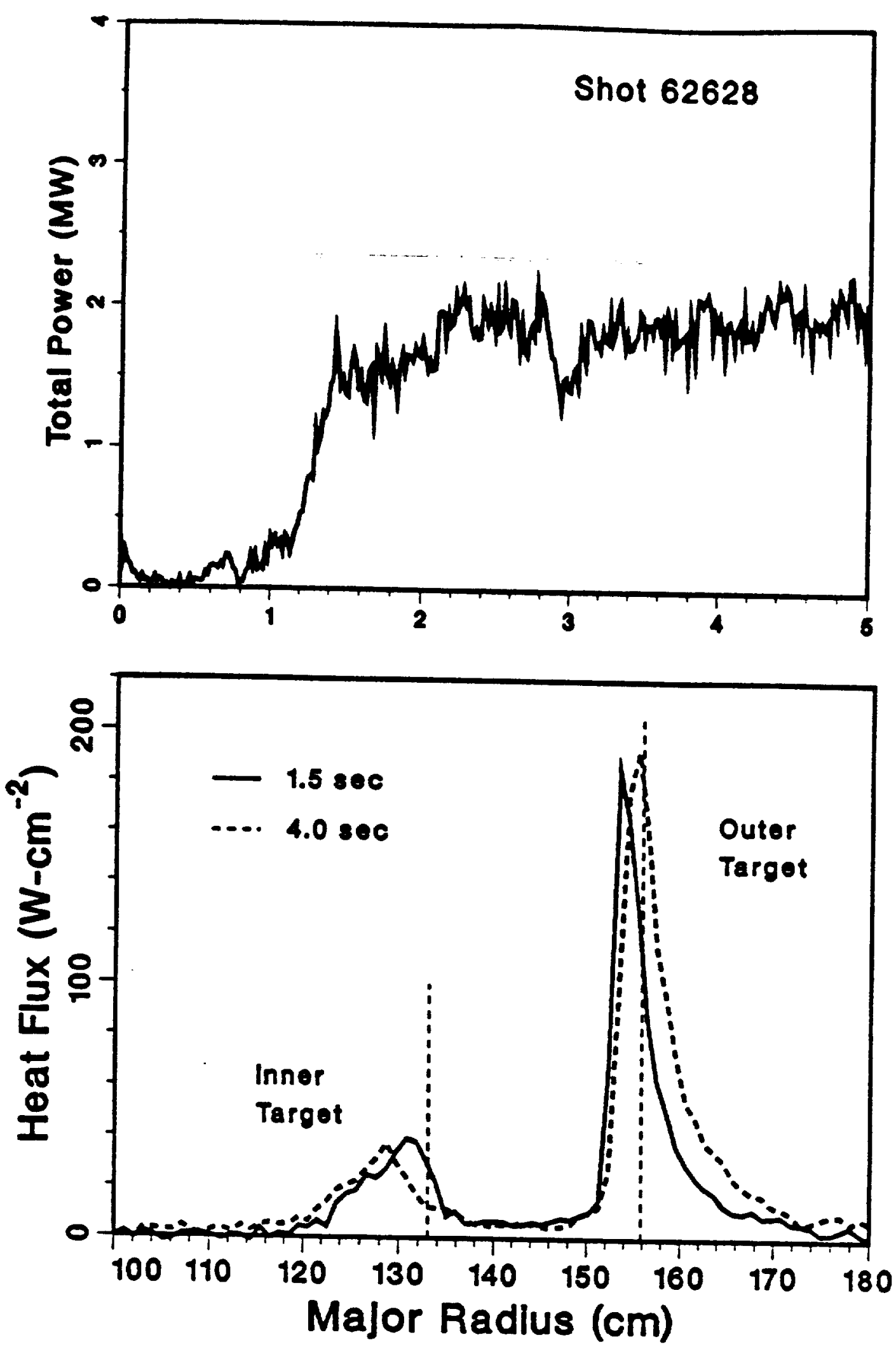

Fig. 5. Divertor heat flux for quasi-stationary H-mode. Top-total divertor power versus time. Bottom, radial profiles at the times indicated. Dashed vertical lines are the inner and outer strike points. 


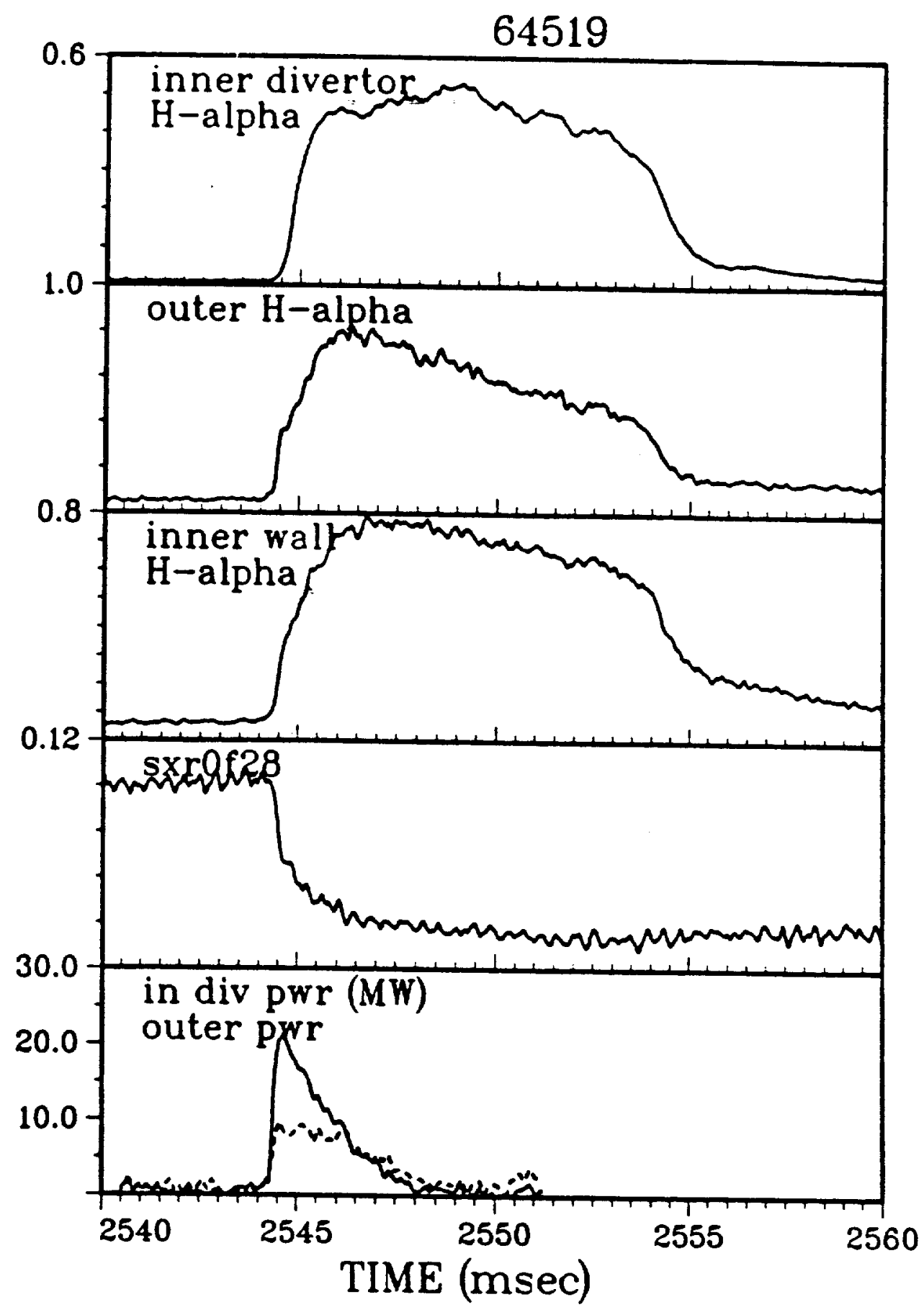

Fig. 6. Divertor heat flux during a typical ELM. Top three traces are divertor $H_{\alpha}$ emission at the inner strike point (PHD01F), X-point (PHD02F), and outer strike point (PHDO4F). Bottom traces show total divertor heat flux at inner and outer strike points in MW. 

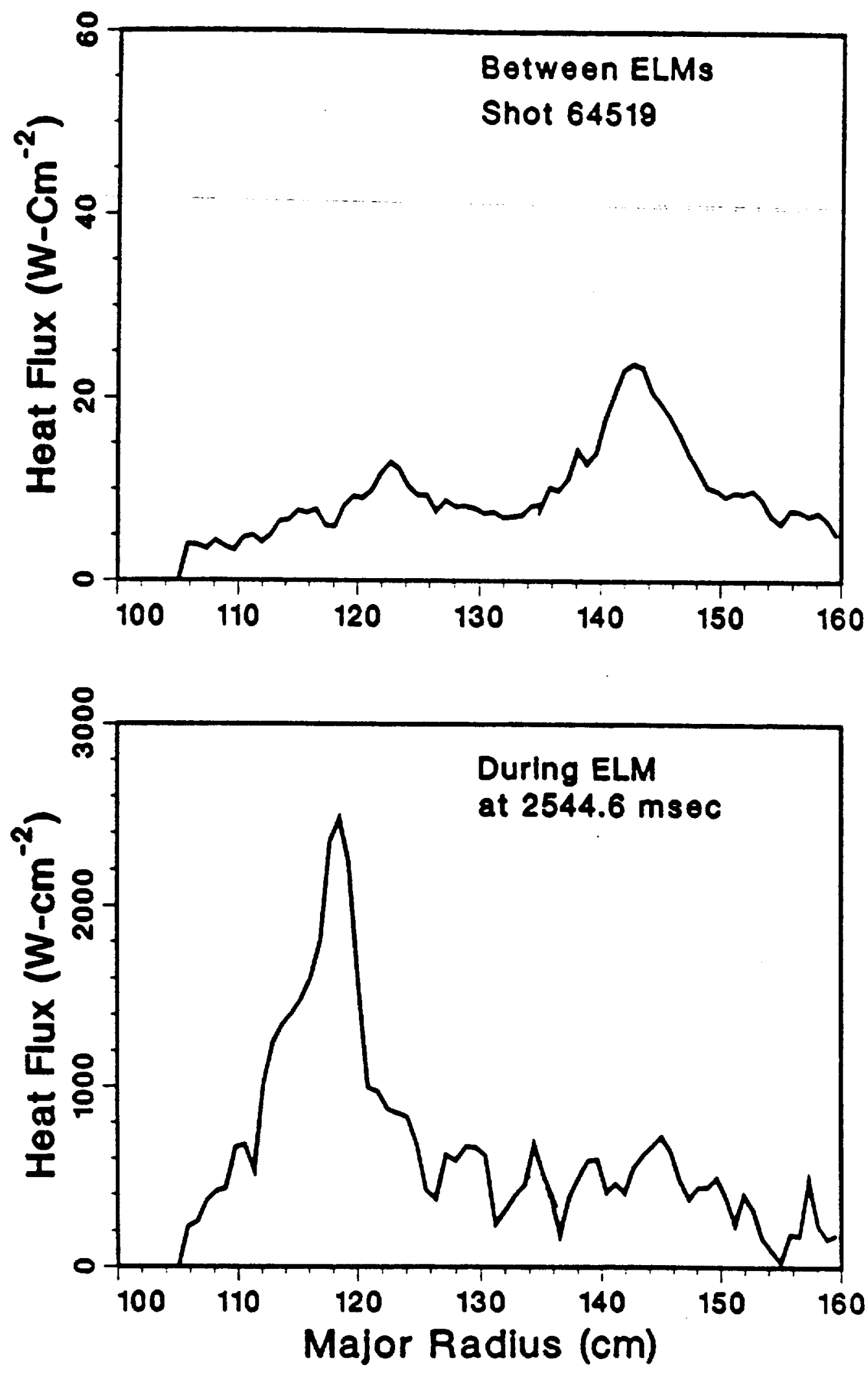

Fig. 7. Radial profiles of divertor heat flux between and during ELMs. 
The large divertor heat pulses due to ELMs should also produce modulation of the local electron temperature and density. With the present electronics we cannot sweep the probe voltage fast enough to follow these changes, but we can follow the change in ion saturation current during an ELM. Furthermore, if the strike point is swept across the probe while ELMs are occuring, we can obtain a complete profile of the divertor ion flux due to ELMs as well as that arriving during the quiescent periods between them. Such data is plotted in Fig. 8, which shows the ion saturation current (top), divertor $\mathrm{H}_{\alpha}$ emission (middle), and major radius of the $\mathrm{X}$-point (bottom) for one such steady-state $\mathrm{H}$-mode discharge. The $\mathrm{H}_{\alpha}$ trace documents the amplitude of the repetitive ELMs during the $\mathrm{H}$-mode. The $\mathrm{X}$-point sweep moves the strike point from outside to inside the probe location so that we can equate time with radial position. The envelope of pulses gives the profile of the divertor ion flux during the ELMs; the peak is about five times higher than the baseline and is situated outside the separatrix intercept. The envelope of the baseline corresponds to the profile of steady losses between the ELMs. We have integrated these two curves to find the relative contribution of the ELMs to the total divertor particle flux and find that the time-averaged loss due to the ELMs is slightly larger than that comming out during the quiescent periods between the ELMs. We are now trying to incorporate these results into our pumping calculations for the divertor pumping experiments.

We have started to look at the effect of ELMs on the global power balance in these steady-state $\mathrm{H}$-mode plasmas. A power balance is constructed using data from a bolometer array, the divertor IR camera, and diagnostics for neutral beam input power and total stored energy. We divide the power losses into three components: radiation, conduction to the divertor between ELMs, and fow to the divertor during ELMs. During the steady state the time-average total stored energy remains constant and so power balance requires $\mathrm{P}_{\mathrm{NBI}}=\mathrm{P}_{\mathrm{rad}}+\mathrm{P}_{\mathrm{div}}+\mathrm{P}_{\mathrm{ELM}} . \mathrm{P}_{\mathrm{ELM}}$ is given by $\Delta \mathrm{W} \cdot f$, where $f$ is the ELM frequency and $\Delta W$ is the energy loss per ELM determined from either MHD analysis or the IR TV data. Table I. and Fig. 9 show these quantities for several $\mathrm{H}$-mode plasmas. Total radiative losses (divertor and core plasma) account for about $30-50 \%$ of the input power. Heat flux to the divertor targets between ELMs accounts for another $30 \%$, while the flux due to ELMs remains nearly constant at $1 \mathrm{MW}$. However, we have not included the increased radiative losses due to the ELMs because we cannot measure such losses on the fast time scale necessary to follow a single event. 


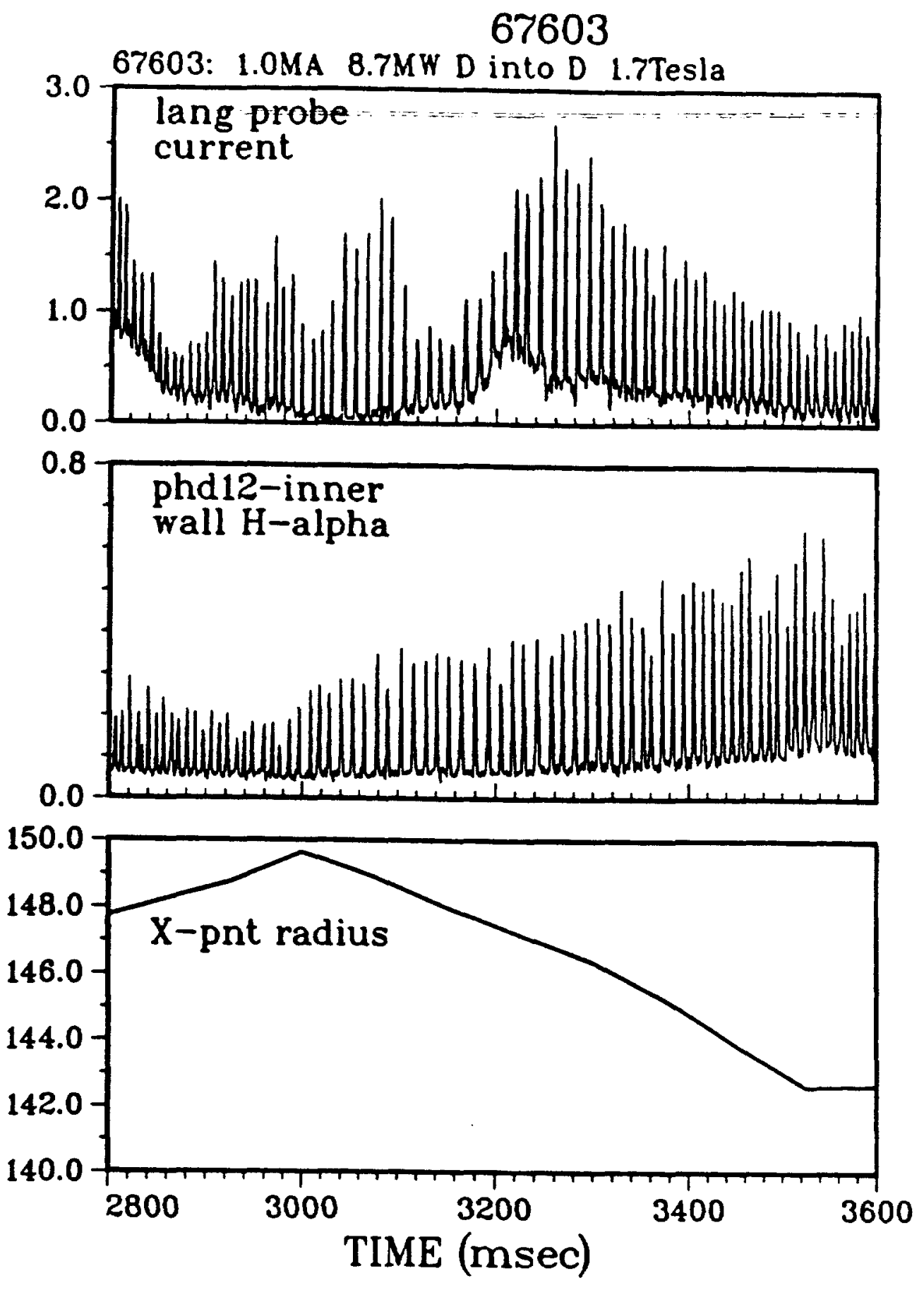

Fig. 8. Radial profiles of divertor particle flux (Langmuir probe ion saturation current), top, $H_{\alpha}$ emission at outside intercept, middle, and program voltage for $X$-point sweep, bottom. 
Table I

POWER BALANCE FOR H-MODES WITH ELMS

Quasi-stationary power balance with ELMs losing $\Delta E$ at a repetition rate $f$ is:

$$
P_{\text {Tot }}=\dot{W}+P_{\text {rad }}+P_{\text {div }}+P_{E L M}^{\text {pb }}
$$

where $P_{\text {Tot }}=P_{N B I}+P_{O H}$, and $P_{\text {div }}$ is the total divertor heat load between $E L M s$, and $P_{E L M}=f \Delta E$.

Shot \# $I_{p} P_{\text {Tot }} P_{N B I} \quad \dot{W} P_{\text {rad }} P_{\text {div }} f_{\text {elm }} P_{\text {elm }}^{\text {dia }} P_{\text {elm }}^{\text {Pir }}$ time (ms)

$\begin{array}{llllllllll}64519 & 1.9 & 2.7 & 1.8 & 0 & 1.5 & 0.65 & 16 & 0.9 & 0.9\end{array}$

03000

$\begin{array}{llllllllll}64519 & 1.9 & 2.7 & 1.8 & 0.83 & 1.9 & 0.2 & 0 & 0 & 0\end{array}$

$\mathbf{Q 2 1 0 0}$

$\begin{array}{llllllllll}64516 & 1.9 & 4.4 & 3.5 & 0 & 2.3 & 1.2 & 15 & 1.0 & \text { NA }\end{array}$

02800

$\begin{array}{llllllllll}64523 & 1.9 & 4.5 & 3.5 & 0 & 2.0 & 1.1 & 32 & 1.4 & 1.76\end{array}$

02600

$\begin{array}{llllllllll}64517 & 1.9 & 6.0 & 5.3 & 0 & 4.0 & 1.0 & 35 & 0.9 & \text { NA }\end{array}$

$\mathbf{0 2 2 5 0}$

$\begin{array}{llllllllll}64510 & 1.9 & 7.5 & 6.3 & 2.2 & 2.8 & 1.2 & 0 & 0 & 0\end{array}$

02000

$\begin{array}{llllllllll}64510 & 1.9 & 7.5 & 6.3 & 0 & 2.7 & 2.1 & 11 & 1.1 & \text { NA }\end{array}$

02400

$\begin{array}{llllllllll}64513 & 1.9 & 7.7 & 6.3 & 0 & 3.0 & 2.1 & 11 & 1.1 & \text { NA }\end{array}$

02400

64434

02500

64520

$\mathbf{0 2 0 5 0}$

64520

$\begin{array}{lllllllll}1.5 & 7.8 & 7.3 & 0 & 2.5 & 2.3 & 57 & 1.2 & \text { NA }\end{array}$

$\mathbf{0 2 7 0 0}$

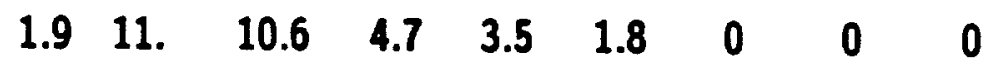

$\begin{array}{lllllllll}1.9 & 11 . & 10.6 & 0 & 3.5 & 3.4 & 61 & 2.5 & \text { NA }\end{array}$ 

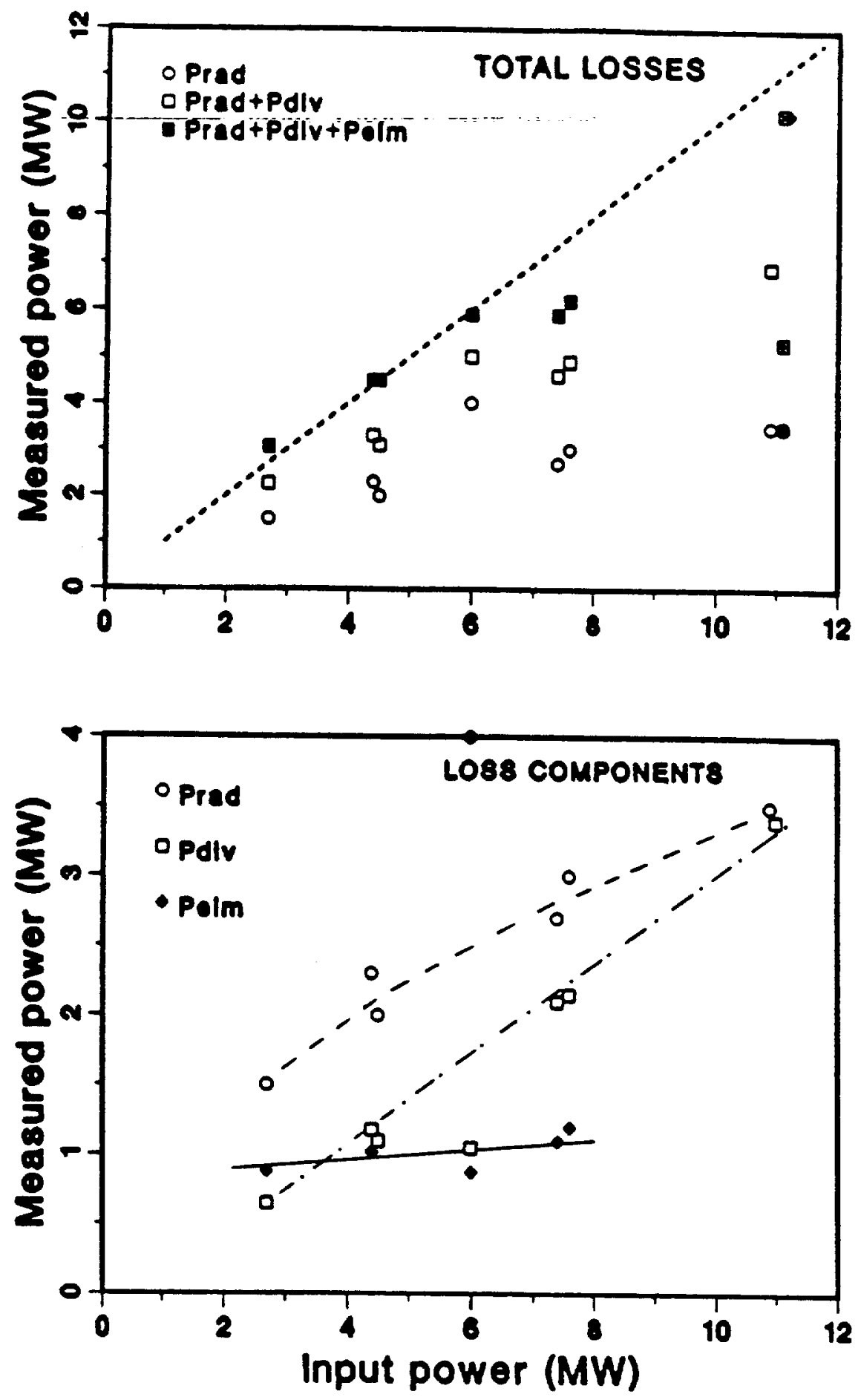

Fig. 9. Power balance for steady-state $\mathrm{H}$-mode discharges. Top-sum of all loss components. Bottom-components plotted separately. 


\section{Divertor Heat-Flux Asymmetries}

Asymmetries in the divertor heat flux have important implications for ITER, since factors of two in local heat loads can mean the difference between having the target plates survive or burn up or erode away. In DIII-D the largest poloidal asymmetries in peak heat flux $\left(P_{\text {out }} / P_{\text {in }} \geq 4\right)$ are recorded for NBI heated L-mode and $\mathrm{H}$-mode plasmas with rapid ELMs; ohmic and ELM-free $\mathrm{H}$-mode discharges have more symmetric power deposition on the divertor targets. L-mode plasmas show the largest inboard/outboard asymmetry (up to factors of six) in total divertor power, $\int 2 \pi R P(R) d R$. In a toroidal device we can expect a minimum heat flux asymmetry due to geometric considerations such as the fact that there is more surface area on the outboard side of the plasma than on the inside. In addition, the MHD equilibrium shift of the finite-beta plasma compresses the outboard magnetic flux surfaces, making steeper radial gradients and increased perpendicular losses there. This is shown in a plot of the calculated energy flux per unit area across the separatrix, Fig. 10, generated by the B2 SOL transport code. Together, these toroidal effects should produce a 2:1 divertor-target heat flux asymmetry in DIII-D, as documented in Table II.

Other mechanisms exist which can increase the divertor heat flu asymmetry. Staebler and Hinton ${ }^{3}$ have shown that poloidal electric fields can produce $\mathrm{E} \times \mathrm{B}$ drifts large enough to influence power flow to the divertor targets. There also may be poloidally localized anomalous losses in the bad curvature region at the outboard midplane. The shorter field-line connection length to the outboard divertor would increase the heat flux there. Both of these mechanisms may be responsible for the large heat-flux asymmetries observed in some L-mode plasmas (see Fig. 11) and in ELMing H-mode plasmas (see Fig. 5).

The change in divertor heat flux profiles with the change in toroidal field direction offers additional evidence that particle drifts may affect power flow out of the core plasma. Fig. 12 shows profiles for the ohmic, L-mode, and $\mathrm{H}$-mode phases of two discharges which have similar parameters $\left(\mathrm{I}_{p}=1.38 \mathrm{MA}, \overline{\mathrm{n}}_{e} \simeq 4 \times 10^{13} \mathrm{~cm}^{-3}\right.$, maximum $6 \mathrm{MW} \mathrm{D}^{\circ} \rightarrow \mathrm{D}^{+},|\mathrm{B}|=1.5$ Tesla $)$, except for the reversal of $\mathrm{B}_{T}$ and a change in $\mathrm{X}$-point position $\left(\mathrm{R}_{x}=145 \mathrm{~cm}, \delta z_{x}=20 \mathrm{~cm}\right.$ for $59984 \mathrm{vs} 135 \mathrm{~cm}$ and $14 \mathrm{~cm}$ for $62758 ; \delta z_{x}$ is the vertical distance from the $\mathrm{X}$-point to the divertor). For 


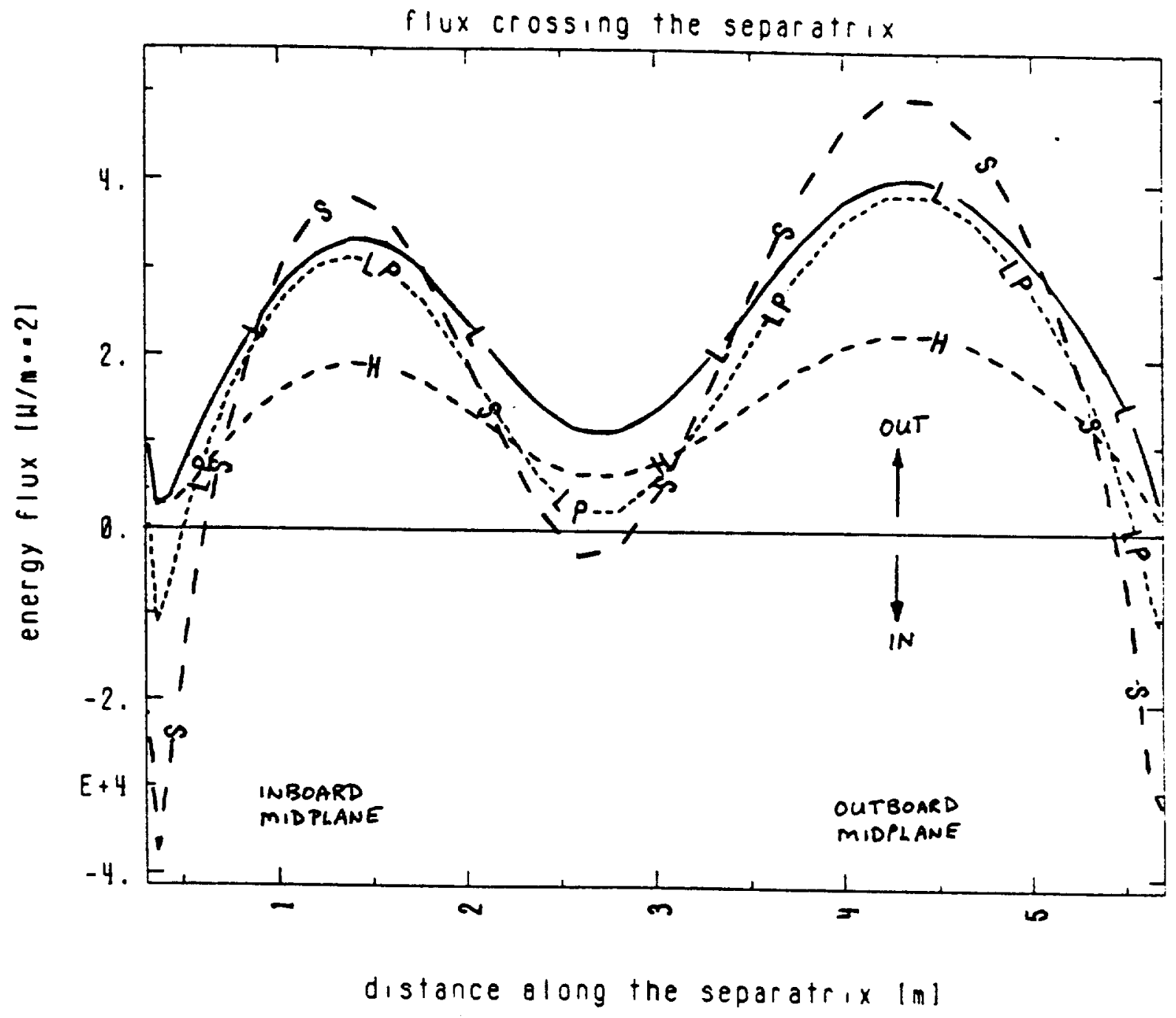

Fig. 10. Energy flux across the separatrix as a function of distance along the separatrix calculated using the B2 code. Labels correspond to transport coefficients of Table II. 
Table II.

\section{HEAT-FLUX ASYMMETRY STUDIES}

All runs have no private region, but $B_{\theta}=0$.

- Effect of radial transport model

\begin{tabular}{|l|l|l|}
\hline Transport & Pout $_{\text {div }} / P_{\text {div }}^{\text {in }}$ & Run \# \\
\hline ITER-L & 1.85 & 7A \\
ITER-LP & 2.29 & $7 B$ \\
ITER-H & 1.76 & $7 \mathrm{G}$ \\
Standard & 2.37 & $7 \mathrm{C}$ \\
\hline \multicolumn{2}{|l}{ Separatrix BC are uniform $n_{e}=1.9 \times 10^{10}$ and $T_{e}=47 \mathrm{eV}}$. \\
\hline
\end{tabular}

- Effect of boundary conditions (ITER-L coefficients)

\begin{tabular}{|l|l|l|l|}
\hline B.C. & Pout $_{\text {div }} /$ Pin $_{\text {div }}^{\text { }}$ & Flux & Run \# \\
\hline$n_{e}=1.9 \times 10^{10}, T_{e}=47 \mathrm{eV}$ & 1.85 & $1260 \mathrm{~A}, 1.4 \mathrm{MW}$ & $7 \mathrm{~A}$ \\
$\Gamma=1.44 \times 10^{20}, \mathrm{~T}_{e}=47 \mathrm{eV}$, & 1.82 & $1260 \mathrm{~A}, 1.3 \mathrm{MW}$ & $8 \mathrm{~A}$ \\
$\Gamma=1.44 \times 10^{20}, Q_{e}=20,300$ & 2.0 & $1260 \mathrm{~A}, 1.4 \mathrm{MW}$ & $8 \mathrm{~J}$ \\
$Q_{i}=5556$ & & & \\
\hline
\end{tabular}




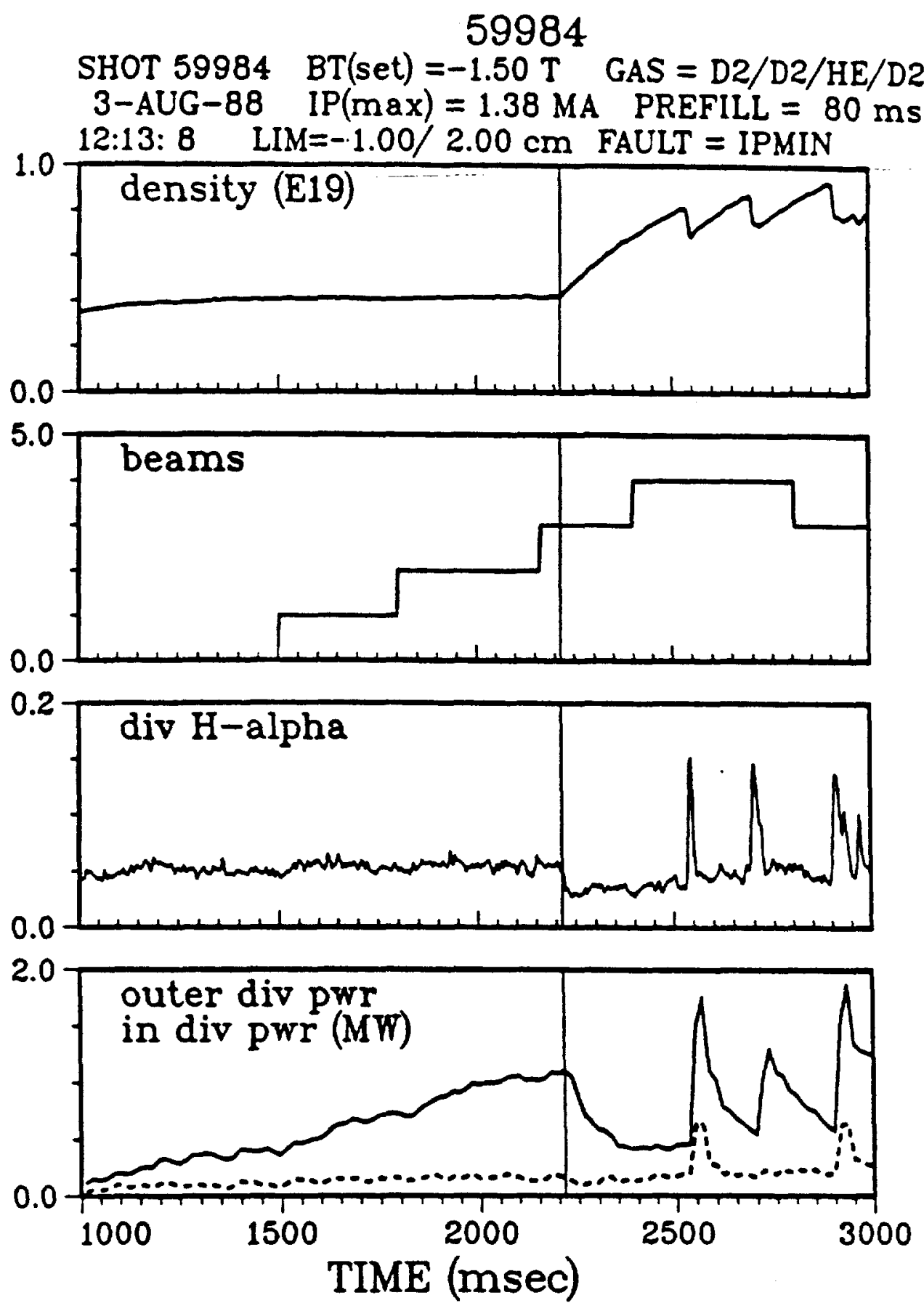

Fig. 11. Typical parameters for $\mathrm{L}$-mode and $\mathrm{H}$-mode discharge showing large divertor heat flux asymmetry. From top to bottom-line density, NBI heating power, divertor $\mathrm{H}_{\alpha}$ emission, and total divertor heat flux at outer strike point (solid) and inner strike point (dashed). 


\section{DIVERTOR HEAT-FLUX PROFILES Reversed/Normal Bt Operation}
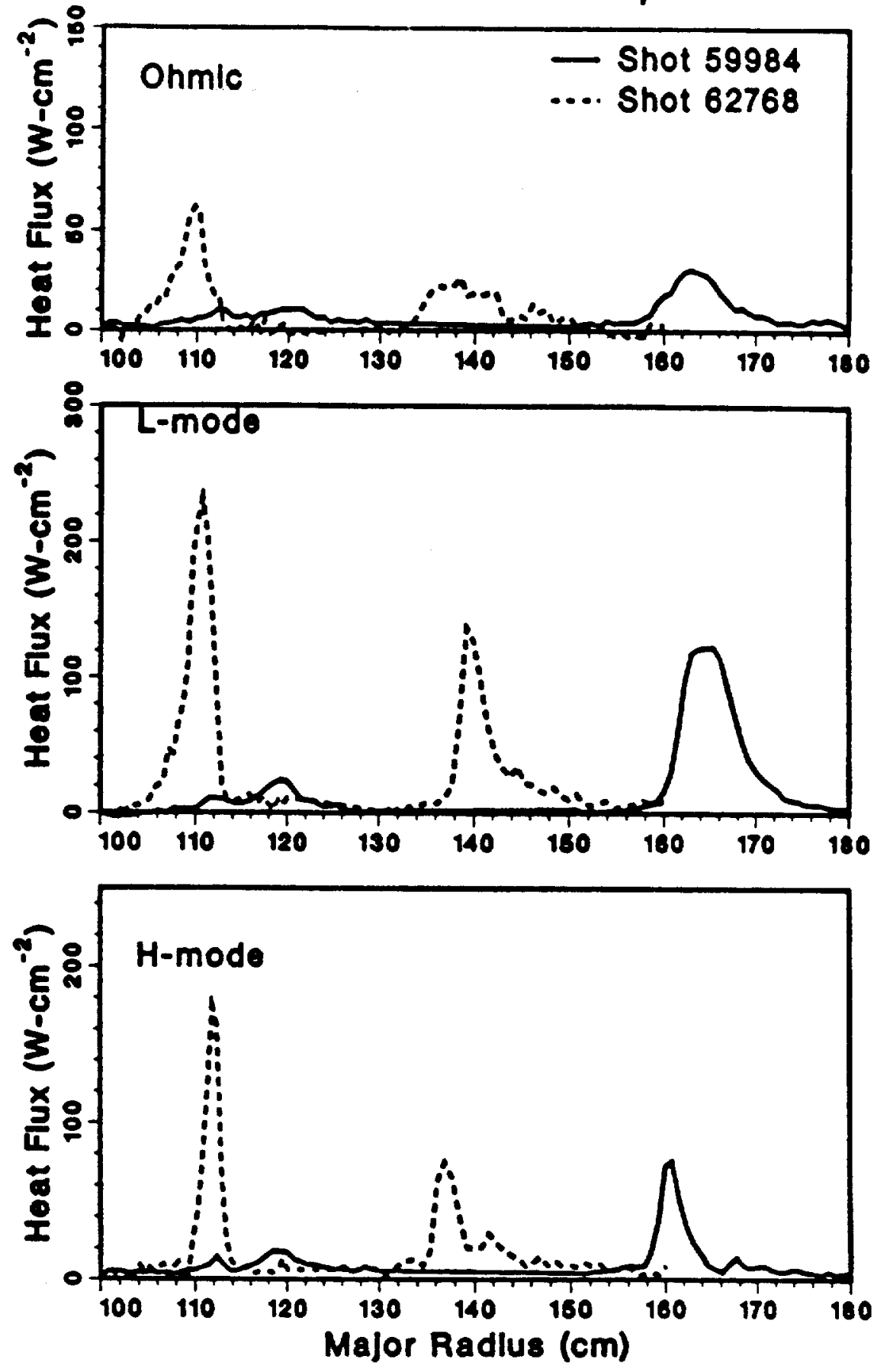

Fig. 12. Divertor heat flux profiles for two similar plasmas except for a reversal of toroidal field so that the ion $\nabla B$ drift is away from (dashed) instead of towards (solid) the $X$-point. Vertical lines correspond to the separatrix intercepts. 
discharge 59984 the ion $\nabla B$ drift is in the direction of the divertor, while for 62758 it is reversed. 2D SOL models without $E \times B$ cannot reproduce this change in asymmetry with field direction. A more complete model ${ }^{19}$ which includes thermoelectric E-field drifts such as those formulated by Staebler and Hinton has successfully reproduced reversed- $\mathrm{B}_{T}$ data from JFT-2M. We hope that the upgrade of the B2 code now in progress will give new results in better agreement with the DIII-D data.

Some heat flux asymmetries have also been observed in double-null divertor discharges, which we now describe. The double-null (DN) configuration should have the advantage of distributing the divertor heat load over a larger area than for a single null discharge. Attaining the maximum benefit from DN operation requires that the two X-points be positioned accurately enough so that the two separatrix flux surfaces are coincident to within the heat-flux e-folding length $(0.5 \mathrm{~cm}$ at the outboard midplane for $\mathrm{H}$-mode discharges in DIII-D ). Otherwise, all the power flow across the innermost separatrix will be conducted along field lines to the one target plate. If this were not possible in a large device such as ITER, then a time-averaged balanced heat load could probably be maintained by oscillating the position of one null relative to the other.

In DIII-D successful double-null operation has been obtained with up/down balanced divertor heat loads and good $\mathrm{H}$-mode confinement. The shaping controls were designed to maintain a balanced magnetic flux at the two nulls while allowing their position to be adjusted relative to the vessel walls; the exact balance was adjusted with a programmable waveform. MHD analysis of the plasma shape showed that the two separatrix surfaces were coincident to within $\pm 0.2 \mathrm{~cm}$ at the outboard midplane The resulting total power to each divertor target $\left(\int 2 \pi R \cdot P(R) d R\right)$ was balanced to within $20 \%$ (the error bar for the power measurements), as shown in Fig. 13(a). Or the other hand, the peak power at the targets was not so well balanced (b). The peak heat flux on the lower target plate (in the ion $\nabla \mathrm{B}$ drift direction) is nearly twice that on the upper target. This may be due to drift effects, but we have no carried out systematic studies to determine how this imbalance varies with toroida field direction or relative position of the two nulls. We have so far only made a crud 
scan of divertor heat flux profiles vs the relative null position, which shows that the power can be transferred from one divertor to the other (Fig. 14) as expected.

\section{L-mode SOL Data and Modeling}

Data from L-mode discharges is very important for SOL plasma model verification because the resulting steady state conditions can be modeled using existing time-independent 2D codes. We are presently constructing a database which contains information on how several key divertor parameters scale with input power, density, current, and magnetic geometry such as X-point height. In this Section we discuss the scaling of the plasma pressure at the separatrix, the divertor heat and particle flux, and the total radiative losses as a function of NBI heating power. Then we present some of the recent results of modeling these plasmas using the Braams B2 code.

\section{A. Scaling Data for L-mode Discharges}

The variation of plasma pressure at the separatrix with input power is an important input needed for modeling the scrape-off-layer in future devices such as CIT or ITER. This results from the fact that present codes obtain the power scaling of divertor parameters by assuming that power crosses the separatrix according to the relationship $\mathrm{Q}_{\perp}=\mathrm{n}_{\mathrm{e}} \chi \nabla_{\perp} \mathrm{T}$ where $\chi$ is independent of power. The power flow across the separatrix then increases with plasma pressure (nT) at the separatrix. It may be, however, that $\chi$ is temperature dependent and should also scale with input power. We have started to examine the variation in $L$-mode edge pressure as a function of input power to see if the present approach is reasonable.

The edge plasma pressure is determined using data from the Thomson scattering system, which measures the density and temperature profiles along a vertical chord passing through the plasma. The position of the sample points relative to the plasma flux surfaces is determined from the ENERGY code and is accurate to within $1 \mathrm{~cm}$ equivalent radius at the midplane. This accuracy is acceptable since in $\mathrm{L}$-mode the edge density and temperature gradients are rather modest near the separatrix. A larger source of error arises from the relative low edge temperature (50 to $100 \mathrm{eV}$ ) which is near the limit of resolution of the system. We show the measured variation of 

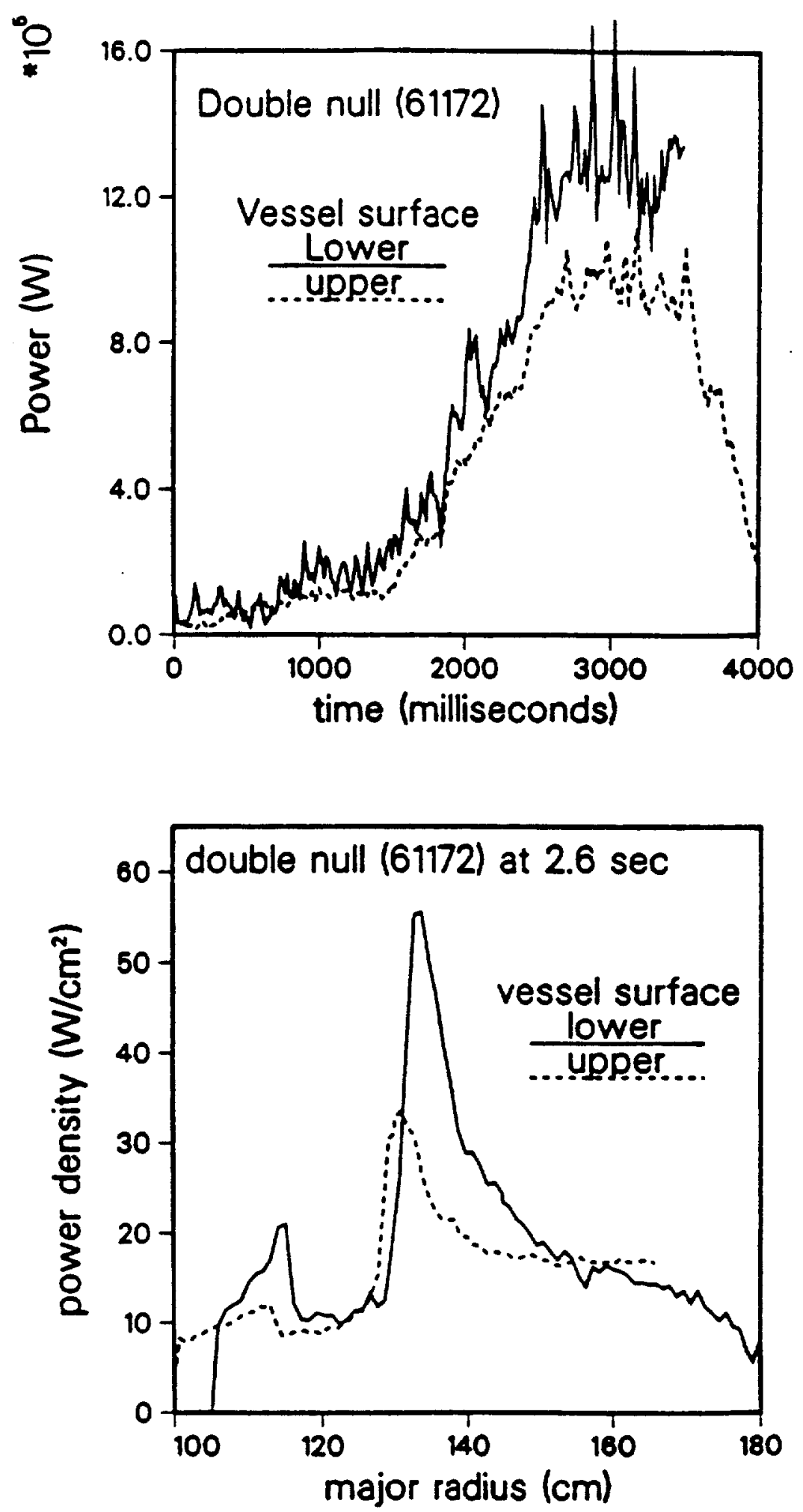

Fig. 13. Divertor heat flux for double-null divertor discharge. (a) Total power to upper and lov divertors. (b) Heat flux profiles at each divertor. 

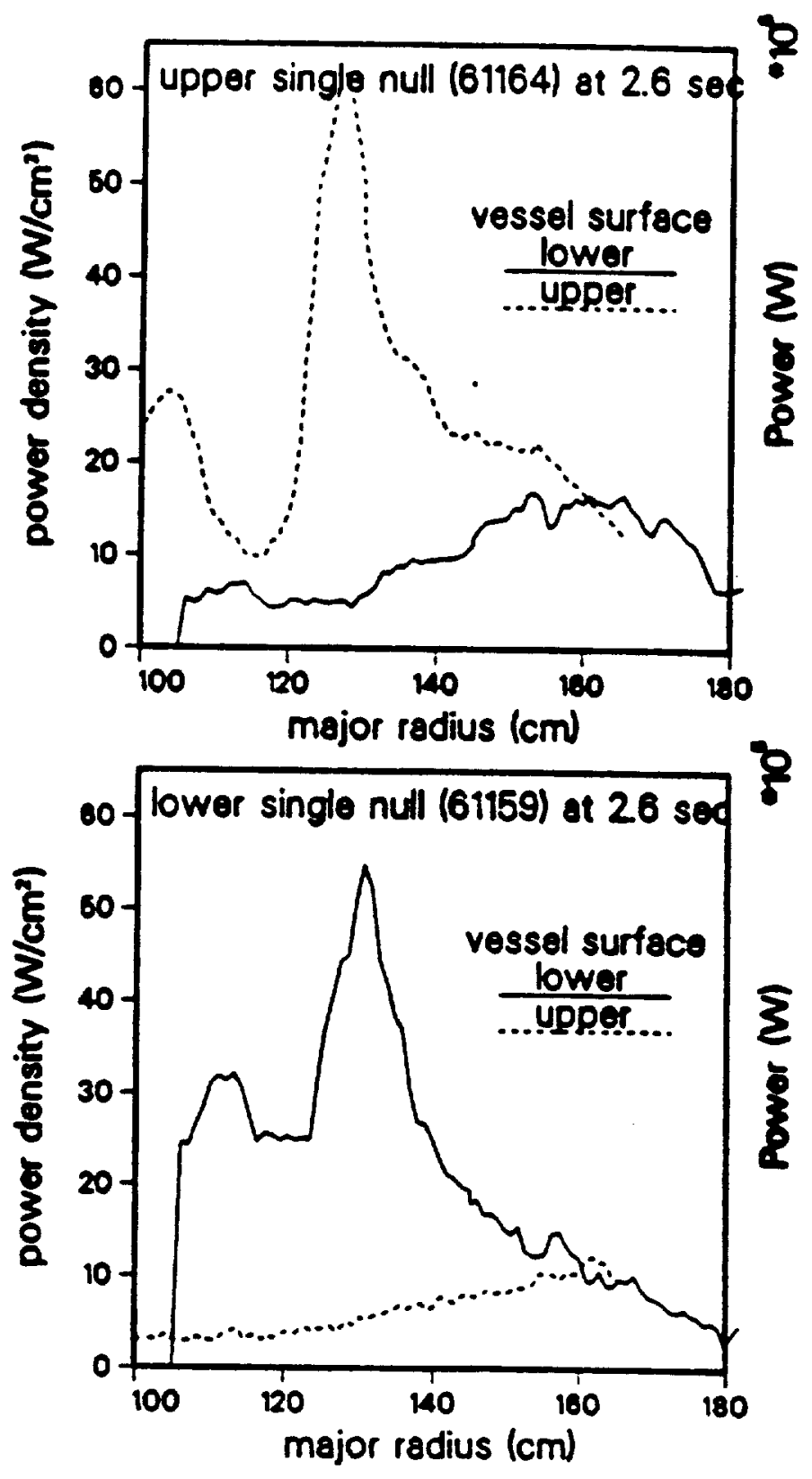

Fig. 14. Divertor heat flux profiles (top and bottom) versus time for discharges with upper and lower single-null configurations. 
edge pressure with input power in Fig. 15; measurements at two different minor radii are included in the data set. The plasma pressure does increase with input power, but the scatter in the data is too large to differentiate between a linear increase or some other power law. We clearly need the better measurements that will be provided by the new reciprocating Langmuir probes to be installed as part of the Advanced Divertor Program.

The fraction of the input power reaching the separatrix also figures into these scaling studies. In DIII-D the radiative losses are measured with a 21 channel bolometer array which views both the divertor and the core plasma. A rough estimate of the fraction of power radiated in the core can be obtained for lower single null discharges by assuming that the losses from inside the separatrix are up/down symmetric and then using only the bolometer data from channels viewing the upper half of the plasma. Figure 16 shows how these radiative losses scale with input power in L-mode discharges. In (a) we plot the fraction of input power crossing the separatrix; only about $15 \%$ is radiated from within the core for $I$-mode. Since the total radiative loss is in the range $25-50 \%$ of the input power, (b), a significant radiative loss must occur in the SOL plasma itself. This fraction, about $20-35 \%$, is plotted in (d). The power which finally reaches the divertor targets (Fig. 17) is approximately $30 \%$ of the input power, which leaves $20-40 \%$ of the losses un-measured.

The installation of new graphite probe tips for two of the divertor Langmuir probes has provided us with new data on the L-mode scaling of the divertor plasma parameters at the outboard strike point. In September we completed a two-day Divertor Characterization Experiment during which we measured the electron temperature and density profiles as a function of plasma current ( 0.8 to $1.6 \mathrm{MA})$, neutral beam heating power ( 1.5 to $6 \mathrm{MW}$ ), and line density $\left(2.5\right.$ to $\left.6 \times 10^{13} \mathrm{~cm}^{-3}\right)$. For L-mode discharges in hydrogen (chosen to increase the $\mathrm{H}$-mode threshold power) we increased the NBI power during the shot in a stepwise manner, and at each power level slowly swept the separatrix out across one of the probe tips and then back in. A typical sequence is shown in Fig. 18, which includes plots of the $\mathrm{X}$-point radial position and the current to the probe tips as a function of time (the rapid modulation of the signal is the result of continuously sweeping the probe bias-voltage to trace out the I-V characteristic). The $\mathrm{X}$-point sweep started $100 \mathrm{msec}$ after each step in beam power, and required $500 \mathrm{msec}$ to shift the divertor strike point from $161 \mathrm{~cm}$ to $172 \mathrm{~cm}(10 \mathrm{~cm}$ inside of the probe to $2 \mathrm{~cm}$ outside). 
Pressure at $r / a=0.90$ from Thomson scattering
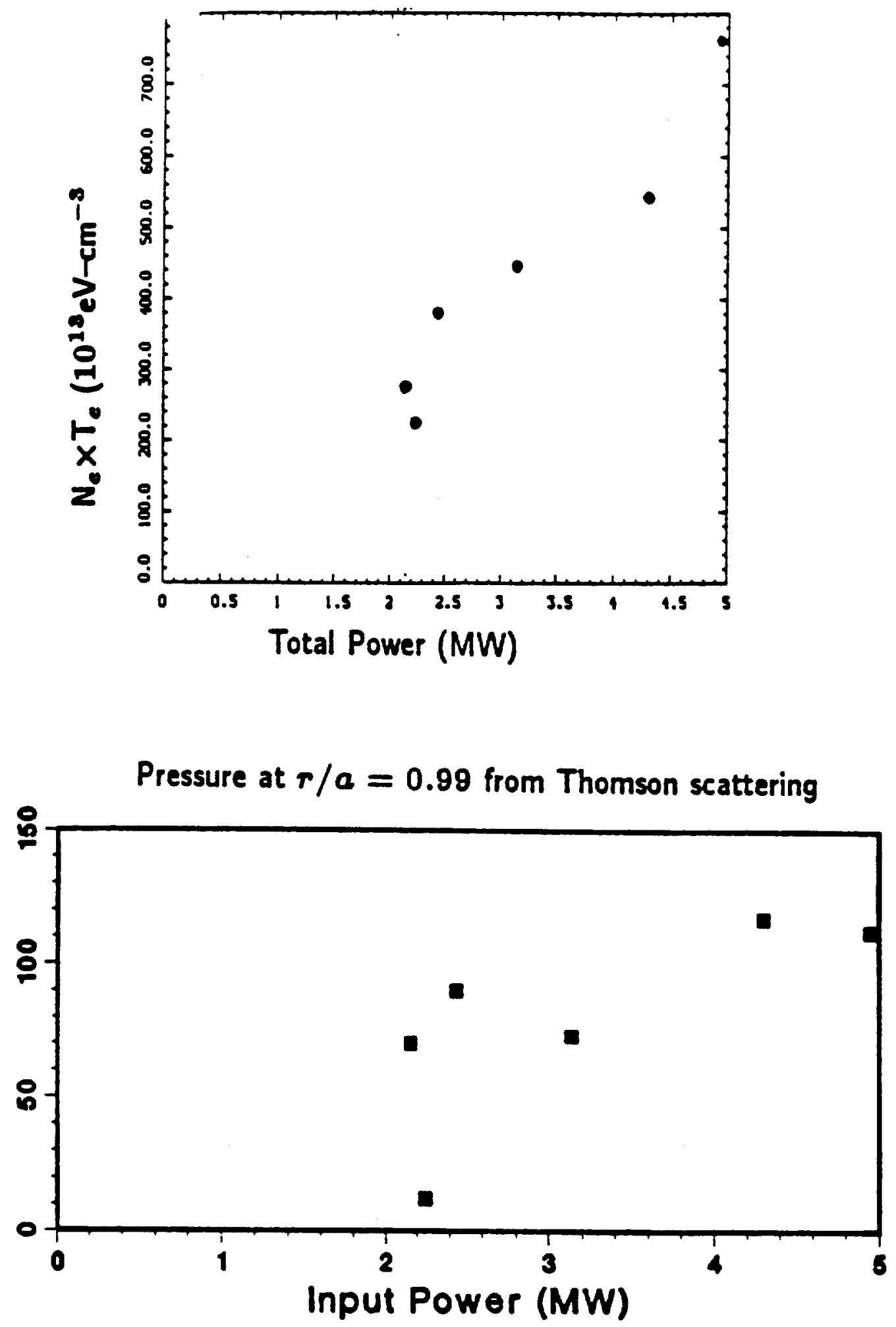

Fig. 15. Variation of edge plasma pressure with NBI heating power for L-mode discharges. 
Fraction of Power Crossing the Separatrix

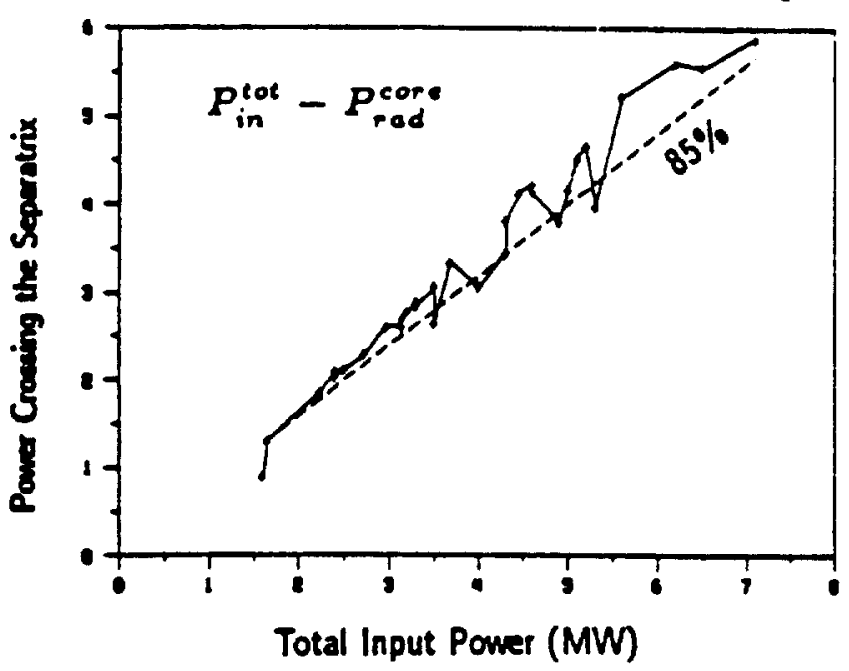

Fraction of Power Radiated Inside Separatrix

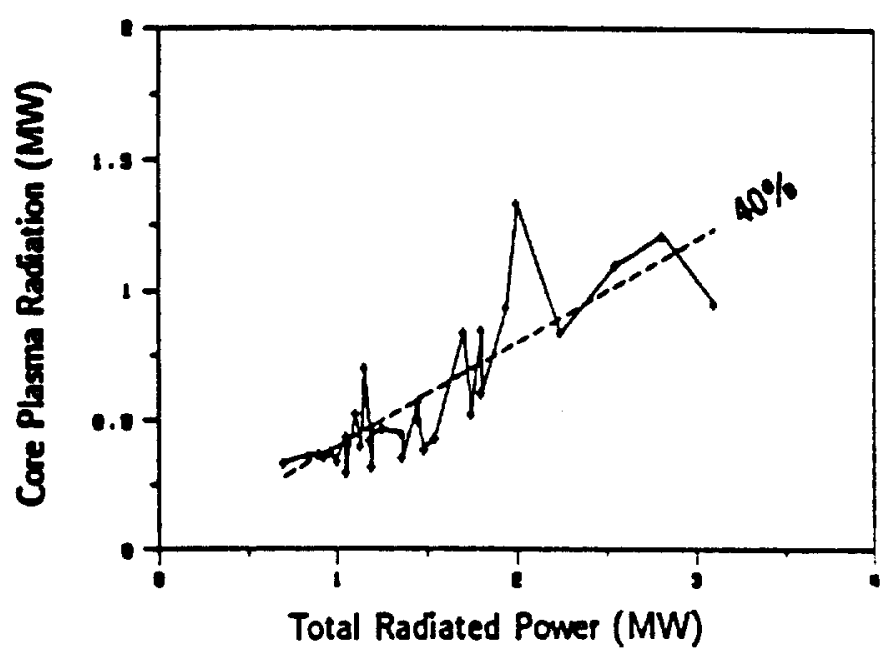

Total Radiated Power (b)

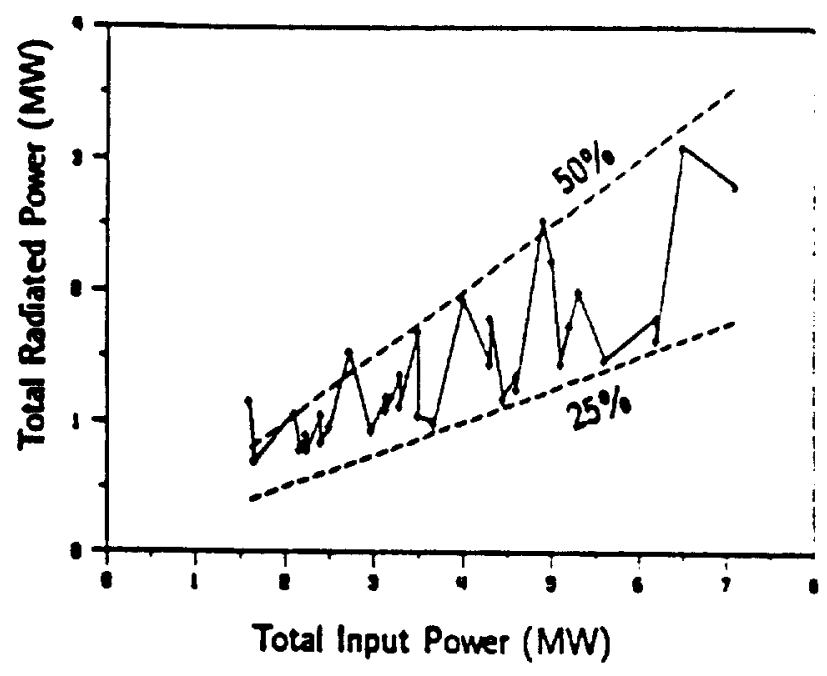

Fraction of Input Power Radiated in the SOL ( $d$ )

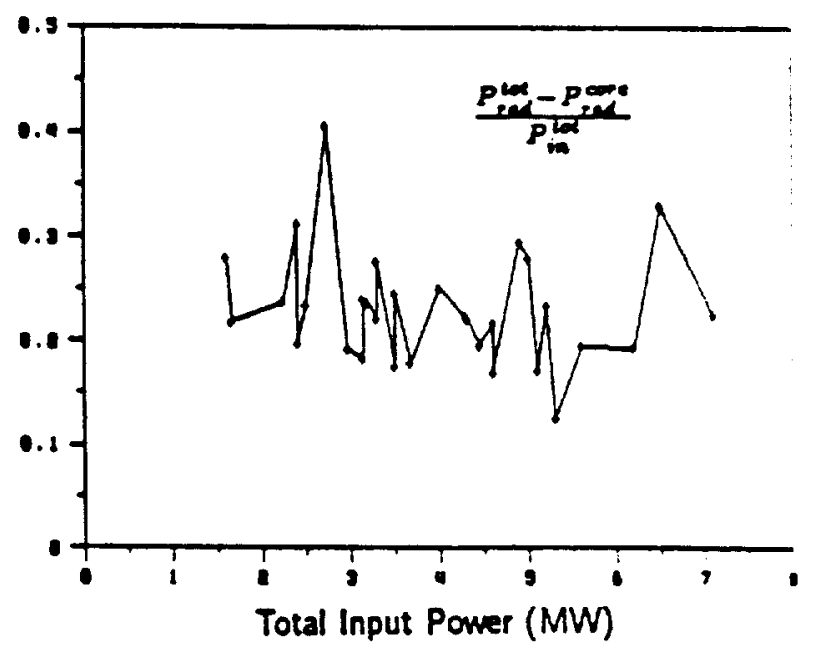

Fig. 16. Radiative losses versus power for $L$-mode discharges. (a) fraction of input power crossing the separatrix (not radiated from inside separatrix). (b) total radiated power as a function of total input power, (c) power radiated from within the separatrix versus total radiated power, (d) fraction of total input power radiated from SOL plasma outside the separatrix. 


\section{L-MODE DIVERTOR POWER}

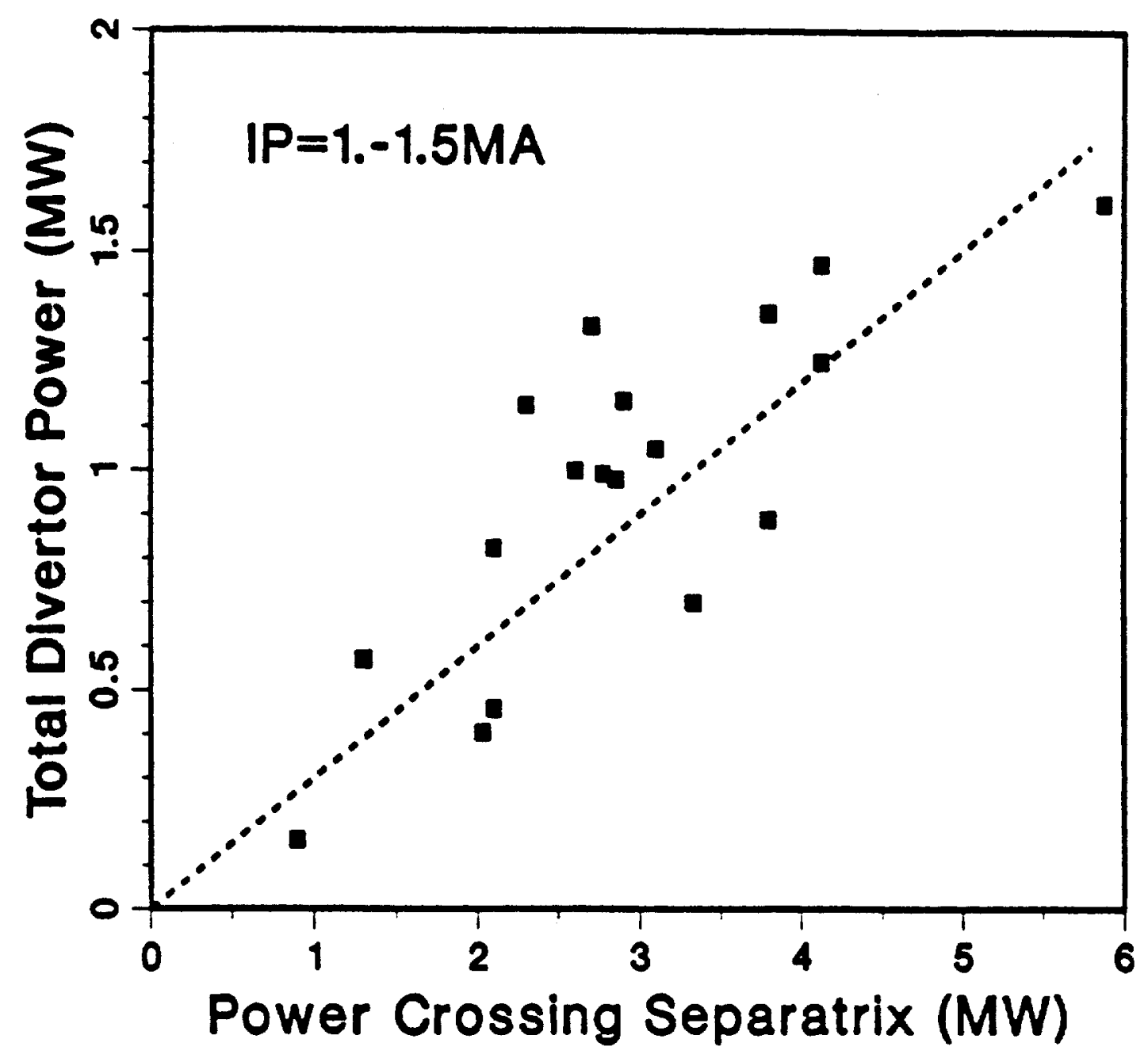

Fig. 17. Total integrated divertor power $\left[\int 2 \pi R \cdot P(R) d R\right]$ versus total input power for $L-$ mode discharges. 


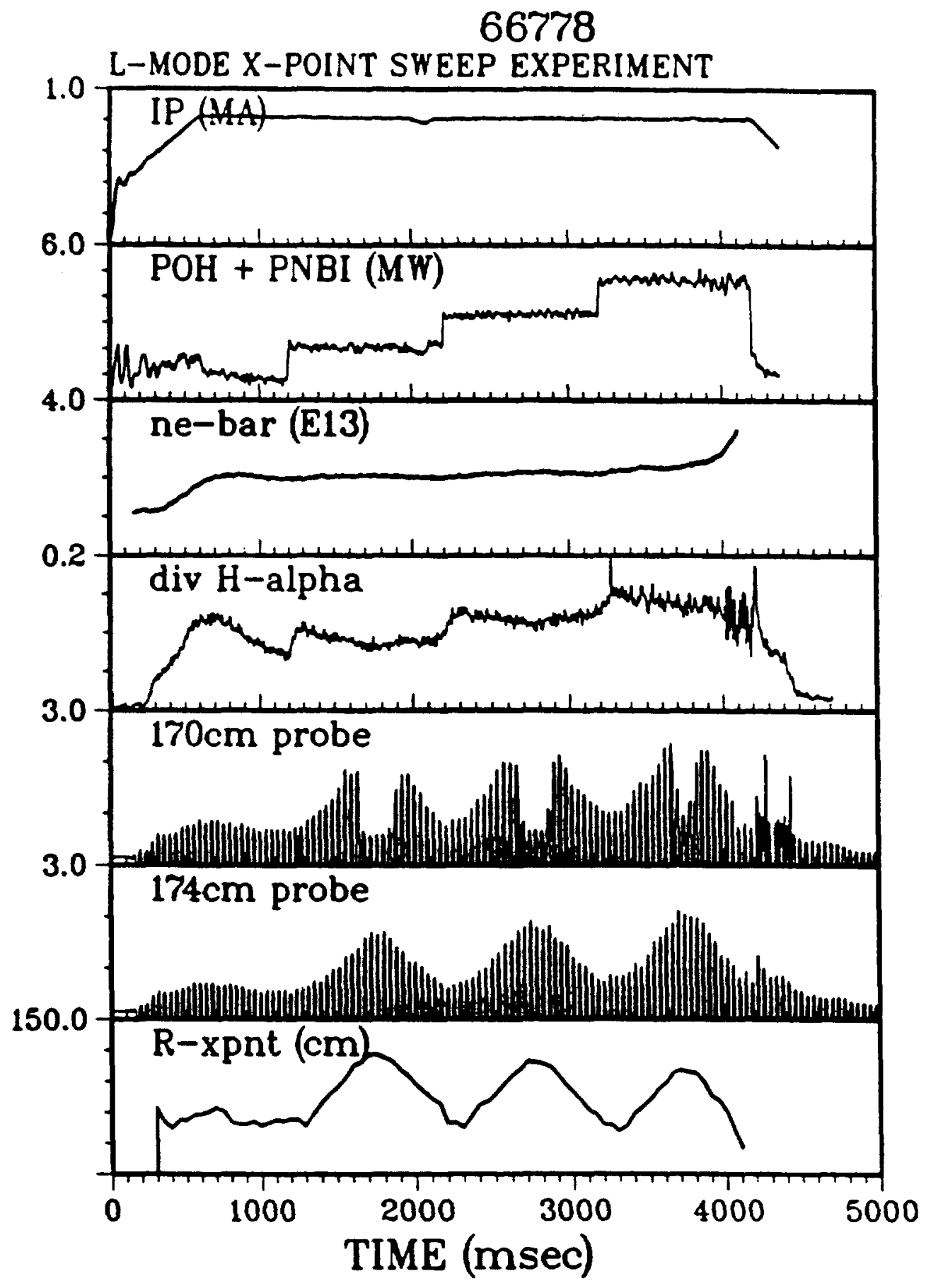

Fig. 18. Discharge characteristics for $L$-mode $X$-point sweep. Top to bottom: plasma current, total input power, line-average, divertor $\mathrm{H}_{\alpha}$ emission, Langmuir probe currents, $X$-point radial position $(\mathrm{cm})$. 
The envelope of the the Langmuir probe signals in Fig. 18 clearly shows the profile of the ion saturation current $\left(\propto n_{e} \cdot T_{e}^{\frac{3}{5}}\right)$ at the outboard divertor target. The ion saturation current (ion flux) increases as the separatrix is moved closer to the probe tips. The sharp reductions in current observed at 1630,2630 , and $3630 \mathrm{msec}$ correspond to times when the separatrix crosses the $170 \mathrm{~cm}$ probe tip; for the next $200 \mathrm{msec}$ or so the tip samples the divertor plasma in the private region, and then, as the separatrix is swept inward back across the probe, the current rises steeply at 1830,2830 , and $3750 \mathrm{msec}$. We thus obtain the two mirror-image divertor plasma profiles shown for each power level.

The measured profiles from the divertor Langmuir probes are in qualitative agreement with our B2 SOL modeling. We find the expected steep gradient across the separatrix, with minimal diffusion into the private fux region (this sets an upper limit on $D_{\perp}$ for the SOL plasma). It appears that the peak ion saturation current $\left(10 \mathrm{~A} / \mathrm{cm}^{2}\right.$ normal to $\left.\mathrm{B}\right)$ does not not change appreciably as the input power increases from 2 to $5 \mathrm{MW}$. Both an analytic ${ }^{20}$ model and the B2 code predict this behavior if the density at the separatrix remains constant; Thomson scattering data taken at each power level shows this to be the case here. Furthermore, these models predict that the electron temperature should rise almost linearly with power, which is consistent with our preliminary analysis of individual I-V traces (25 to $45 \mathrm{eV}$ over this power range). Finally, we see that the area under the curves, which is proportional to the total ion flux to the outboard divertor, is independent of the input power. If the fraction of recycled neutrals that fuels the core plasma remains fixed, then this result would imply that the particle confinement time, $\tau_{p}$, is independent of power. This conjecture, however, requires further neutral transport modeling with DEGAS in order to be substantiated.

\section{B. Modeling the L-mode SOL Plasma Using B2}

We have taken our $\mathrm{L}$-mode data and incorporated it into our modeling of scrapeoff-layer transport using the B2 fluid code. We had several objectives in mind: 1) to determine the sensitivity of the code results to approximations of the actual SOL geometry, 2) to arrive at a set of transport coefficients that gave results in agreement with plasma measurements, and 3) to test the scaling capability of the code by generating output that could be checked easily against experiment. The first two are discussed more fully below, while the last has been covered previously in Section II. 
In the future, we plan to include data from the new divertor Langmuir probes in our benchmarking efforts while work on several upgrades to the code is in progress.

The B2 code approximates the actual DIII-D scrape-off-layer geometry using a rectangular grid with metric coefficients for each cell. The metric takes into account the pitch angle of the field lines as well as the approximate expansion of the flux surfaces near the $\mathrm{X}$-point. However, as usually run, the poloidal field does not vanish and diffusion across the separatrix into the private region is not allowed. We thought that the vanishing poloidal field would lower the peak divertor heat flux due to the logarithmic growth of the field-line connection length near the null. Similarly, we expected that allowing for diffusion into the private region would yield broader heat flux profiles with lower peak values. We therefore modified the B2 magnetic geometry and included the private region in the model. Fig. 19 contains some of the results obtained with these different versions of the code.

The effect of the poloidal field null turns out to be negligible, as shown in Fig. 19(a). This may be explained as follows. The longer connection length can only reduce the heat flux to the divertor if cross-field transport carries it to other flux surfaces. However, the magnetic flux expansion near the $\mathrm{X}$-point reduces the radial density and temperature gradients by the same amount as the connection length grows, so that the ratio of parallel to perpendicular heat flow remains unchanged.

On the other hand, allowing diffusion into the private region has a large effect on the L-mode divertor heat flux profiles, as shown in Fig. 19(b). For this run we used the same diffusion coefficient and convective velocity on the separatrix from the midplane all the way to the divertor targets. This lowered the peak heat flux by almost a factor of two, and shifted it from being on the separatrix to about $1 \mathrm{~cm}$ outside, in better agreement with the L-mode experimental data. Note that changing the cross field transport in the private region could have a large effect on the divertor heat flux profile, but there are presently no data or models to suggest how it might vary in this region.

The sensitivity of the predicted scrape-off-layer parameters to the choice of transport coefficients is shown in Fig. 20. Here we plot four different predictions of the midplane density profile in DIII-D based on B2 runs using values of $D_{\perp}, \chi$, and $v_{\text {conv }}$ listed in Table III. The boundary conditions for the runs had fixed density and temperature on the separatrix, a recycling coefficient of 0.97 at the divertor, and zero 


\section{EFFECT OF THE POLOIDAL FELONULL ON THE DIVERTOR HEAT FLUX}

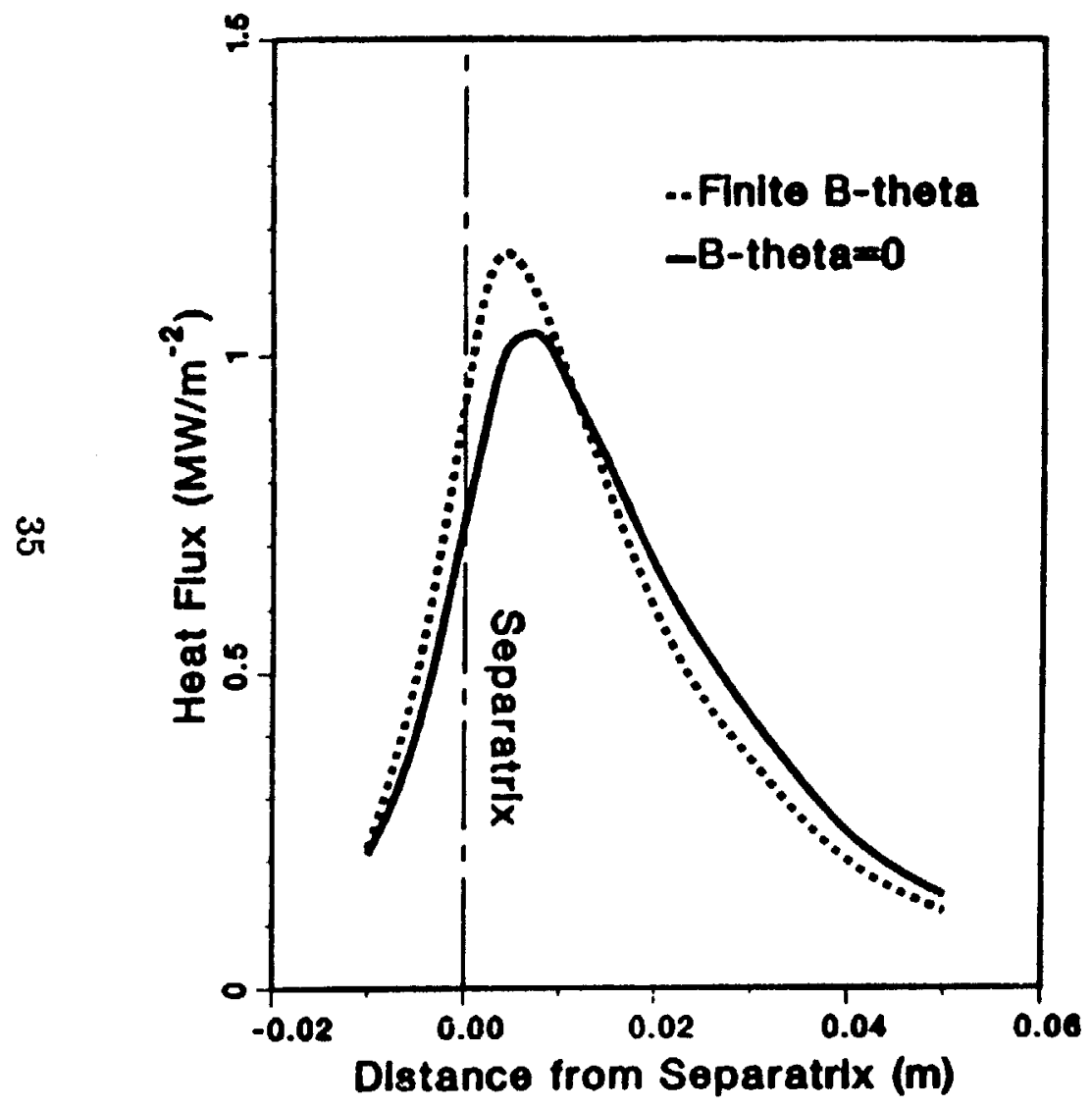

Fig. 19. Outboard divertor heat flux profiles calculated using the B2 code. (a) dashed curve is for non-zero poloidal field at the null, solid has true poloidal field null. (b) dashed curve has no diffusion into the private region, solid does.

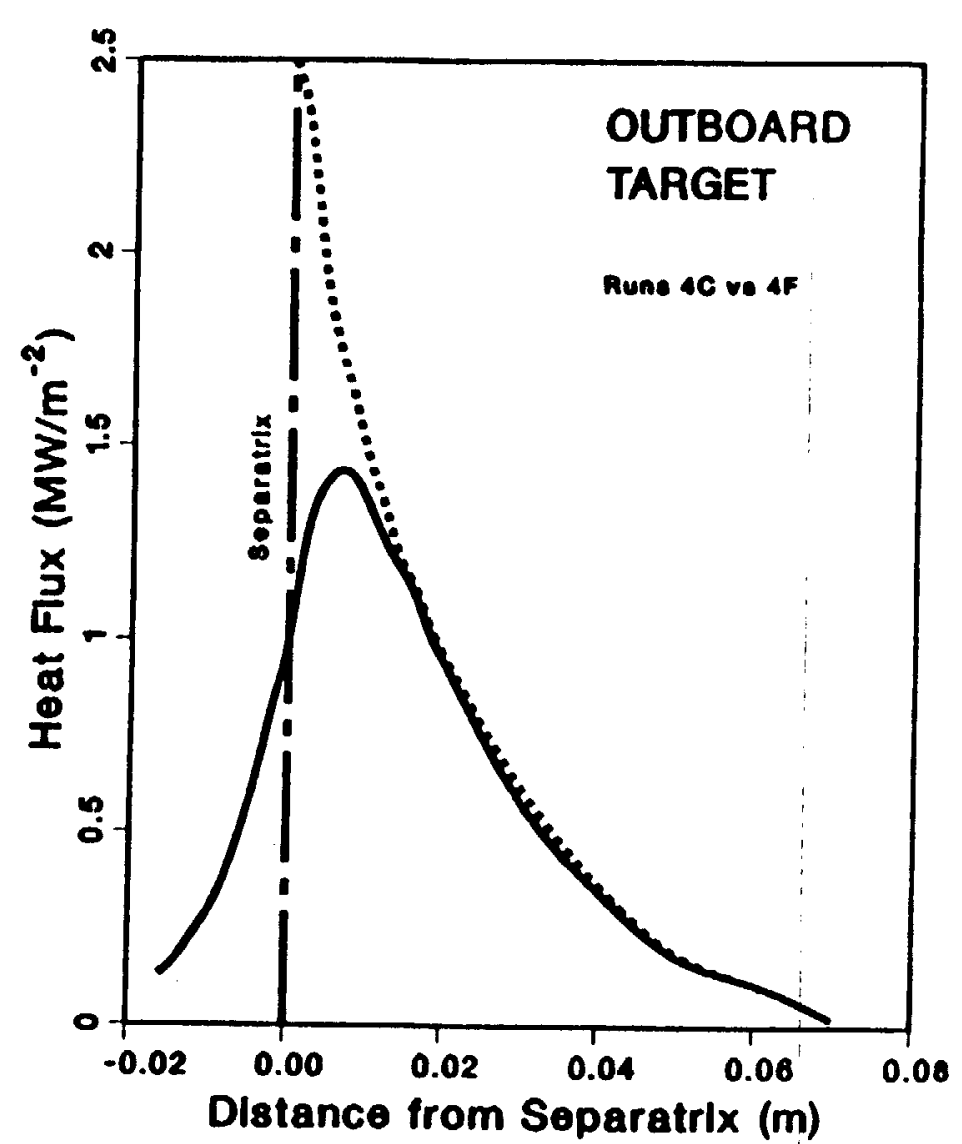

EFFECT OF THE PRIVATE REGION ON THE DIVERTOR HEAT FLUX 


\section{OUTBOARD MIDPLANE DENSITY PROFILES}

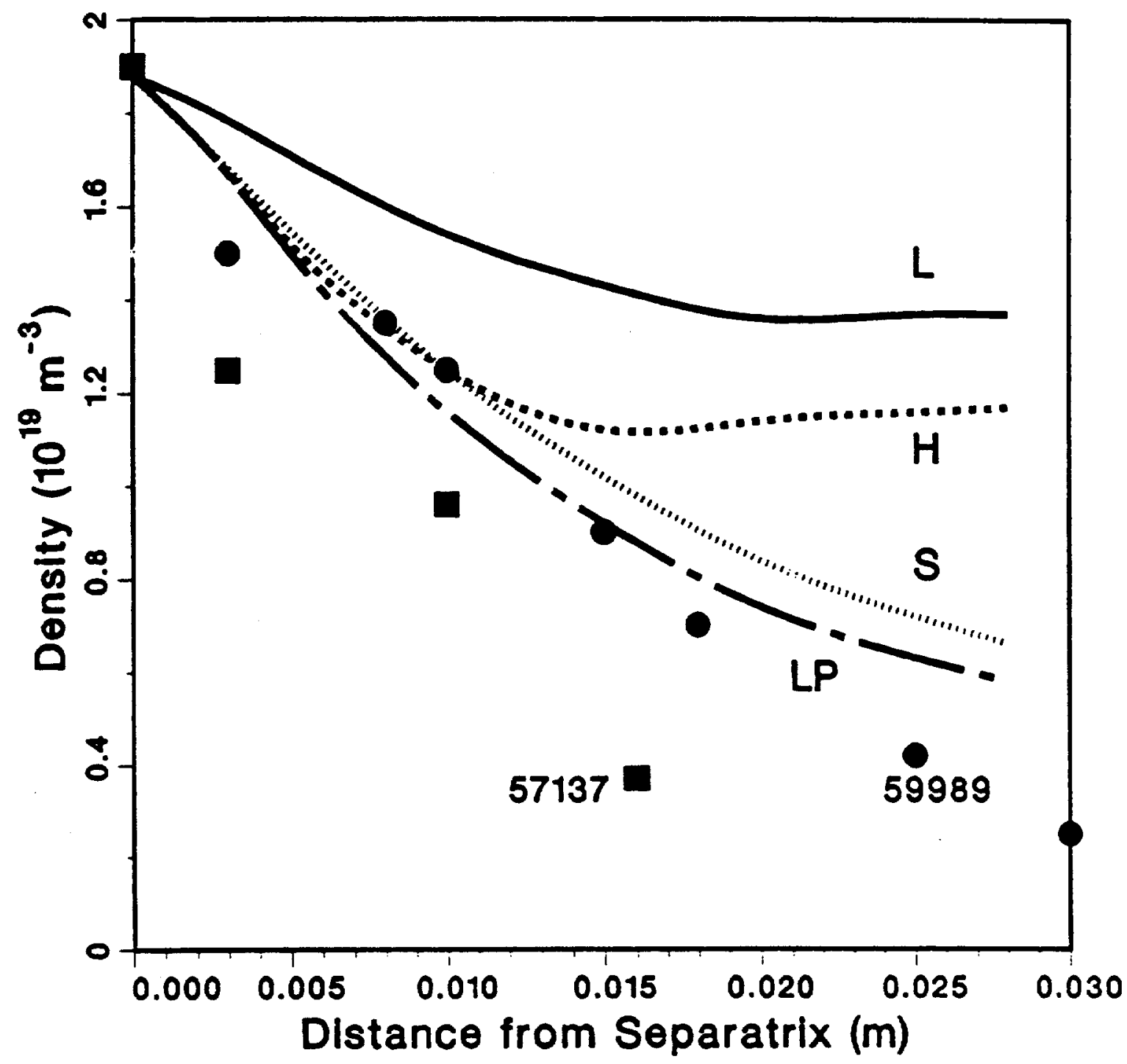

Fig. 20. Comparison of calculated versus measured (solid points) midplane density profiles. Labels refer to transport coefficients of Table II. 


\section{TRANSPORT COEFFICIENTS FOR L-MODE PLASMAS}

We have used four sets of transport coefficients to model the two L-mode plasmas:

Standard ITER-LP ITER-L ITER-H

$\begin{array}{lllll}\chi_{e}\left(\mathrm{~m}^{2} / \mathrm{s}\right) & 4.0 & 3.0 & 3.0 & 1.0 \\ \chi_{i}\left(\mathrm{~m}^{2} / \mathrm{s}\right) & 0.2 & 1.0 & 1.0 & 0.3 \\ \operatorname{difni}\left(\mathrm{m}^{2} / \mathrm{s}\right) & 2.0 & 1.0 & 1.0 & 0.3 \\ \operatorname{vconv}(\mathrm{m} / \mathrm{s}) & -40.0 & -20.0 & 0.0 & 0.0\end{array}$

The following boundary conditions were imposed:

a. At the wall, zero particle flux and $T_{e}=2 \mathrm{eV}$.

b. At the separatrix, $T_{e}=47 \mathrm{eV}$ and $n_{e}=1.9 \times 10^{18} \mathrm{~m}^{-3}$.

The magnetic geometry does not include the "private" flux region, but it does have a vanishing poloidal field near the X-point [ or $B_{p}(\psi)$ ] and flow to both divertor targets is allowed. 
radial particle flux at the wall (located at $0.03 \mathrm{~m}$ in the figure). The scattered points on the plot are measured profiles from two L-mode plasmas. The best agreement is obtained using models with the inward pinch term included; both the ITER-L and ITER-H coefficients give a predicted density profile that is much broader than the measured one, but this may be sensitive to the boundary conditions.

The scaling capability of the code was tested in a rather simple, but direct way. Once we had a set of transport coefficients which described the SOL plasma profile, we varied the energy flux across the separatrix and calculated the resulting inboard/outboard heat flux asymmetry. As discussed in Section III, the B2 code predicts a lower heat flux asymmetry than measured for a wide range of input parameters (see Fig. 11 and Table II). More significantly, the calculated asymmetry is independent of the input power, in sharp disagreement with the experimental data. This implies that the standard B2 code will not yield the correct power scaling of other divertor parameters (e.g., $n_{e}$ and $T_{e}$ ). This is why we attach a lot of importance to upgrading the code with the inclusion of $\mathbf{E} \times \mathbf{B}$ drifts, which are driven by electron temperature gradients and thus may be sensitive to the input power.

\section{Summary and Near-Term Plans}

We have reported here the recent results of our effort to assemble an edge/divertorplasma database to be used as a benchmark for our B2 modeling of the SOL plasma. We have also reported some of the results of the B2 simulations and identified areas where the code needs significant upgrading to better agree with the data. Essentially, we have:

- Measured the divertor-plasma density and temperature profiles for ohmic, $\mathrm{L}$-mode, and H-mode plasmas. Peak L-mode temperatures of $40-50 \mathrm{eV}$ were measured with $5 \mathrm{MW}$ of input power; this drops by about $30 \%$ following the $\mathrm{H}$-mode transition. The peak density was about $3 \times 10^{19} \mathrm{~m}^{-3}$.

- Measured the overall power balance and divertor-plasma parameters for steady-state $\mathrm{H}$-mode discharges. We find that about $30 \%$ of the input power reaches the divertor targets during the quiescent periods between ELMs; radiative losses account for another $30 \%$. The ELMs themselves produce a smaller time-averaged heat flux which is nearly independent 
of input power. The ELM's contribution to the time-averaged divertor particle flux is about equal to the steady flux measured during the quiescent periods.

- Compared the measured inboard/outboard heat flux asymmetry with that predicted by the B2 code and found the model does not yield the observed power and magnetic field dependence. Therefore, we are modifying the code to include $\mathbf{E} \times \mathbf{B}$ drifts.

- Measured the up/down and inboard/outboard heat flux asymmetries for double-null discharges and found that, while we did balance the total power flow to the top and bottom divertor regions, the peak heat flux on the bottom divertor (in the ion $\nabla B$ drift direction) was almost a factor of two higher than that on the top.

- Measured the power scaling of the divertor parameters for L-mode discharges and found that, as in the steady-state $\mathrm{H}$-mode case, about $30 \%$ of the input power is deposited on the divertor targets. We find that a comparable fraction is radiated away in the SOL itself, from which we concluded that radiation cooling of the divertor plasma is a significant process for determining the target-plate heat flux and electron temperature.

- Compared the measured midplane density and divertor heat flux profiles with those predicted by the B2 code using the ITER reference case transport coefficients. The calculations obtained broader density profiles than measured. We also found that it was necessary to include diffusion into the private flux region to produce divertor heat flux profiles similar to those measured in DIII-D .

In the near term, we will be working to expand the experimental database, upgrade the B2 code, and further benchmark B2 against new data. Expansion of the database is already underway as we analyze more divertor Langmuir probe data from the two working prototypes and as we work to install the full poloidal array during the December vent. We are now working on quantifying how the peak electron temperature and density scale with input power and plasma current. These data will provide another benchmark for B2 power scaling studies. We are also planning to analyze Thomson scattering data to better determine how the edge pressure gradient varies with power, from which we can infer how the transport coefficients should scale. 
Several upgrades of the B2 code are now in progress. First, Braams is changing the neutral transport model to better approximate the two-dimensional flow of neutrals away from the divertor targets. We are adding the ability to model non-zero parallel current flow, which can produce significant changes in the sheath potential at the divertor targets. This could affect the observed heat flux asymmetries by changing the electron conduction across the sheaths. Over the longer term, we are working to add $\mathbf{E} \times \mathbf{B}$ drift effects in B2. This should give us the observed dependence on toroidal field direction lacking in the present version. It may also explain the inward shift of the peak heat flux following the $\mathrm{H}$-mode transition.

As we get new data and upgrade the code, we will continue to compare the computational results to the experimental data. We especially want to check the predicted scaling of $T_{e, d i v}$ and $n_{e, d i v}$ at the inner strike point using the data from the new probe tips. We also want to explore other means for producing the observed heat flux asymmetries, such as using spatially varying transport coefficients to produce anomalous losses peaked at the outboard plasma midplane. We hope that including such effects will give us a version of B2 which can simulate the DIII-D results so that we have increased confidence in its use for CIT or ITER. 


\section{References}

1. B.J. Braams, "A Multi-fluid Code for Simulation of the Edge Plasma in Tokamaks", Net Report NR. 68 (1987)

2. Hill, D.N., Ellis R., Ferguson, W., Perkins, D.E., Petrie, T.W., and Baxi, C., Rev. Sci. Instrum. 59 (1988) 1878.

3. Staebler, G.M., and Hinton, F.L., General Atomics Report GA-A19516 (1989).

4. Staebler, G.M., "Model for Improved Divertor Confinement", General Atomics Report GA-A19796 November 1989.

5. St. John, H., Jahns, G.L., Burrell, K.H., DeBoo, J.C., Bull. Am. Phys. Soc. 32 7V4 (1987) 1897.

6. Harbour, P. J., Summers, D.D.R., Clement, S., Coad, J.P., et al., J. Nucl. Mater. 162-164 (1989) 236.

7. Mahdavi, M. A., Schaffer, Mioduszewski, P., et al., "The DIII-D Collaborative Advanced Divertor Program", General Atomics Report GA-A19547 Debruary 1989.

8. Schissel, D. P., Burrell, K.H., DeBoo, J.C., Groebner, R.J, Kellman, A.G., et al., Nucl. Fusion 29 (1989) 185.

9. Pfeiffer, W.W., Davidson, R.H., Miller, R.L., and Waltz, R.E., General Atomics Report GA-A16178 (1980).

10. Allen, S.L., Rensink, M.E., Hill, D.N., Perkins, D.E., Jackson, G.L., et al., J. Nucl. Mater. 162-164 (1989) 80.

11. Neuhauser, J., Proceedings of the H-mode Workshop at General Atomics, San Diego, CA (1987).

12. Hill, D.N., Petrie, T.W., Mahdavi, M. Ali, Lao, L., and Howl, W., Nucl. Fusion 28 (1988) 902.

13. Stambaugh, R.D. and Lao, L., General Atomics Report GA-D17220 (1983).

14. Stott., P., Bull. Amer. Phys. Soc., 33 (1989) 2 F6.

15. Stambaugh, R.D., Proc. European Physical Soc. (1989). 
16. Gohil, P., Mahdavi, M. Ali, et al., Phys. Rev. Lett.. 61 (1988) 1603.

17. Lao, L., Chu, M.S., Ozeki, T., et al., General Atomics Report GA-A19247 (1988).

18. Ozeki, T., Chu, M.S., Lao, L., Taylor, T.S., Chance, M.S., et al., General Atomics Report GA-A19495 (1989).

19. Ueda, N., Itoh, K., Itoh, S., Tanaka, M., et al., J. Nucl. Mater. 162-164 (1989) 607.

20. Mahdavi, M. Ali, et al., Phys. Rev. Lett.. 47 (1988) 1062. 


\section{Acknowledgements}

We would like to thank R. Ellis of LLNL for maintaining the IR TV and helping to analyze data from it, P. Harbour and L. De Kock of JET for supplying the basic Langmuir probe design, and John Smith of General Atomics for working with us to install the two prototype Langmuir probe tips.

This work was supported by the U.S. Department of Energy under Contract Nos. DE-AC03-89ER51114, W-7405-ENG-48, and DE-AC04-76-DP-00789. 


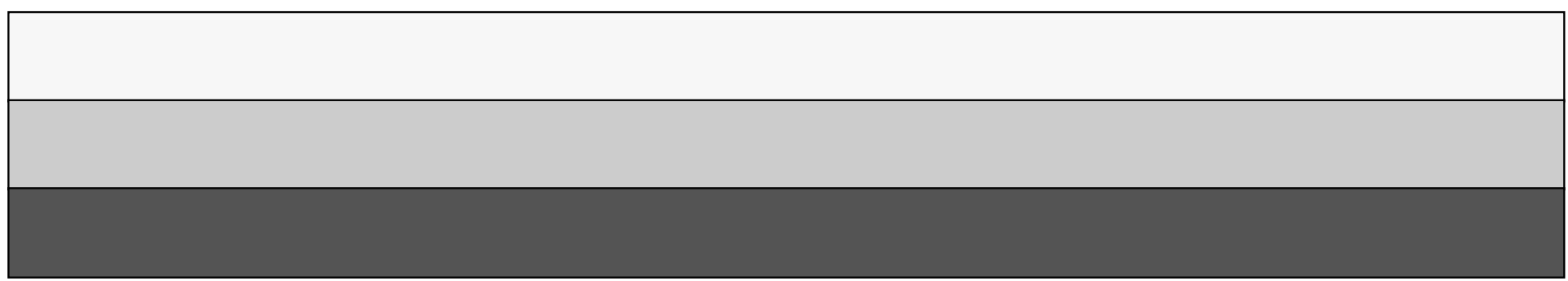

